Title:

\title{
Mitotic binding of Esrrb marks key regulatory regions
}

\section{of the pluripotency network.}

\author{
Authors: \\ Nicola Festuccia ${ }^{1}$, Agnès Dubois ${ }^{1}$, Sandrine Vandormael-Pournin ${ }^{2}$, Elena Gallego Tejeda ${ }^{1}$, Adrien \\ Mouren $^{1}$, Sylvain Bessonnard ${ }^{2}$, Florian Mueller ${ }^{3}$, Caroline Proux ${ }^{4}$, Michel Cohen-Tannoudji ${ }^{2}$ and Pablo \\ Navarro $^{1^{*}}$
}

Affiliations:

1: Epigenetics of Stem Cells, Department of Developmental \& Stem Cell Biology, Institut Pasteur, CNRS UMR 3738, 25 rue du docteur Roux, 75015 Paris, France

2: Mouse Functional Genetics, Department of Developmental \& Stem Cell Biology, Institut Pasteur, CNRS UMR 3738, 25 rue du docteur Roux, 75015 Paris, France

3: Imaging and Modelling, Department of Cell Biology \& Infections, Institut Pasteur, CNRS UMR 3691, 25 rue du docteur Roux, Paris 75015, France

4: Transcriptome and EpiGenome, BioMics, Center for Innovation and Technological Research, Institut Pasteur, 28 rue du docteur Roux, 75015 Paris, France

* Corresponding author: pnavarro@pasteur.fr 


\begin{abstract}
Pluripotent mouse Embryonic Stem (ES) cells maintain their identity throughout virtually infinite cell divisions. This phenomenon, referred to as self-renewal, depends on a network of sequence-specific transcription factors (TFs) and requires daughter cells to accurately reproduce the gene expression pattern of the mother. However, dramatic chromosomal changes take place in mitosis, generally leading to the eviction of TFs from chromatin. Here, we report that Esrrb, a major pluripotency TF, remains bound to key regulatory regions during mitosis. We show that mitotic Esrrb binding is highly dynamic, driven by specific recognition of its DNA binding motif and is associated with early transcriptional activation of target genes after completion of mitosis. These results indicate that Esrrb may act as a mitotic bookmarking factor, opening a new perspective to molecularly understand the role of sequence-specific TFs in the epigenetic control of self-renewal, pluripotency and genome reprogramming.
\end{abstract}




\section{Introduction}

Embryonic Stem (ES) cell lines are derived from the pre-implantation blastocyst and can be maintained indefinitely in culture in an undifferentiated and pluripotent state ${ }^{1}$. Despite undergoing virtually infinite divisions, ES cells do not lose their biological identity nor do they compromise their developmental potential. This phenomenon, known as self-renewal, is strictly dependent upon a network of Transcription Factors (TFs) centred on Oct4, Sox2, Nanog and Esrrb ${ }^{2,3}$. Although these factors act collaboratively ${ }^{3}$, they also display unique functions. While Nanog ${ }^{-1-}$ cells exhibit low selfrenewal efficiency ${ }^{4}$, Oct $^{-/}$or Sox $2^{-/}$undifferentiated cells cannot be maintained ${ }^{5-7}$ and pure undifferentiated $\mathrm{Esrrb}^{-/}$cultures can be propagated only when differentiation is inhibited ${ }^{8,9}$. Moreover, whereas Oct4 and Sox2 up-regulation triggers the loss of pluripotency ${ }^{6,10}$, that of Nanog and Esrrb abolishes the ability of ES cells to differentiate ${ }^{8,11}$. Therefore, even though Oct4 is perhaps the only obligatory and general regulator of pluripotency, the function of Esrrb and Nanog is more specifically dedicated to the control of self-renewal.

ES cells are highly proliferative and present an atypical cell cycle structure characterised by a short G1 phase lacking a G1/S checkpoint ${ }^{12-15}$. Therefore, ES cells effectively exist only in two continuously alternating states of DNA replication and cell division. Generally, mitosis is accompanied by the eviction of TFs and global transcriptional silencing ${ }^{16}$. Given that pluripotency TFs are permanently required to promote self-renewal and prevent differentiation, their activity must be promptly reinstated after each division. Moreover, the inactivation of major chromatin regulators associated with the epigenetic inheritance of gene expression programmes does not have severe detrimental effects on the propagation of the undifferentiated state ${ }^{17-20}$. This large independence from classical epigenetic pathways has recently been recognised as one of the most intriguing hallmarks of ES cells ${ }^{21}$, raising a major unsolved question: how do ES cells maintain the TF-based regulatory continuity from mother to daughter cells and preserve their transcriptional status over indefinite periods of frequent and rapid divisions? 
Increasing observations suggest that some transcriptional regulators can resist chromatin condensation and remain bound to their targets during mitosis. This process, known as mitotic bookmarking ${ }^{22}$, has been reported for general TFs ${ }^{23}$, coactivators ${ }^{24}$ and chromatin modifiers ${ }^{25}$, but also for sequence specific TFs such as Runx $2^{26}, \mathrm{Hnf}^{27}{ }^{27}$, FoxA $1^{28}$ and Gata $1^{29}$. Therefore, mitotic bookmarking by one or more pluripotency TFs could propagate efficiently the regulatory information required to maintain the transcriptional identity of ES cells.

Given the intimate connection existing between their expression and the efficiency of selfrenewal, we initially sought to determine whether Nanog or Esrrb could act as mitotic bookmarking factors. Using fluorescence microscopy we demonstrate that Esrrb, but not Nanog, is retained on mitotic chromosomes, both in ES cells and during early embryogenesis. Furthermore, we report the compendium of genomic locations bound by Esrrb during mitosis, and demonstrate that mitotic Esrrb retention relies on dynamic interactions with its cognate DNA binding motif. Finally, we identify the genes that are either rapidly activated or kept silent after mitosis in response to Esrrb activity. Altogether, we propose that Esrrb may act as a mitotic bookmarking factor in ES cells. 


\section{Results}

\section{Esrrb decorates the mitotic chromosomes in ES cells and early embryos.}

To study whether Nanog and/or Esrrb are retained on mitotic chromosomes, we generated two cell lines expressing either Nanog-GFP or Esrrb-GFP fusion proteins. A Nanog-GFP expression vector was stably integrated into wild-type E14Tg2a ES cells; Esrrb-GFP was introduced in EKOiE ES cells, in which the two endogenous Esrrb alleles were previously deleted and Esrrb is expressed from a Dox-inducible transgene (Methods and Supplementary Fig. 1a online). After confirming that the transgenic GFP-fusions and Dox-inducible Esrrb proteins are functional (Supplementary Fig. 1b online), we monitored their mitotic behaviour. While Esrrb-GFP was systematically localised to mitotic chromosomes, Nanog-GFP was excluded (Fig. 1a, Supplementary Fig. 2a, b online). We then established an immunofluorescence protocol on metaphase spreads prepared from EKOiE cells cultured with Dox. We found that Esrrb was clearly associated to the metaphasic chromosomes (Fig. 1b). We did not observe any signal when Dox was withdrawn for $48 \mathrm{~h}$, demonstrating the specificity of this assay (Supplementary Fig. $2 \mathrm{~d}$ online). Moreover, a similar coating of the chromosomes was observed in metaphase spreads prepared from EKOiE cells expressing an Esrrb Snap-tagged variant, further supporting our observations (Supplementary Fig. 2e online).

To exclude that the mitotic retention of Esrrb is due to the high levels of expression obtained either by Dox induction or by the CAG-driven Esrrb-GFP or Esrrb-Snap transgenes, we fused both endogenous Esrrb alleles to tdTomato (Methods online). We found that Esrrb-tdTomato decorates the chromosomes throughout all phases of mitosis (Fig. 1c, Supplementary Fig. 2c online), as also observed in time-lapse imaging of Esrrb-GFP (Supplementary Movie online). Moreover, when we performed immunofluorescence for Nanog and Esrrb using metaphase spreads prepared from E14Tg2a cells, we observed that Esrrb, but not Nanog, decorates the mitotic chromosomes in wild-type cells (Supplementary Fig. $2 f$ online). Overall, we have documented the chromosomal retention of Esrrb in all mitotic ES cells observed so far using diverse microscopy tools. 
Next, since Esrrb mRNA is abundant in fertilised oocytes and is expressed during early embryogenesis ${ }^{30}$, we asked whether the mitotic retention of Esrrb also occurs in vivo when pluripotency is established. To do this, in vitro transcribed Esrrb-TdTomato and GFP mRNAs were coinjected in single blastomeres of 2 cell embryos. The embryos were let to develop in vitro for $48 \mathrm{~h}$, reaching the compacted morula stage. We observed that Esrrb-TdTomato coats the mitotic chromosomes of GFP positive cells (Fig. 1d). This indicates that the mitotic retention of Esrrb is operational during early embryogenesis.

\section{Mitotic Esrrb is specifically enriched at individual genomic regions.}

To determine whether Esrrb is enriched at specific genomic regions during mitosis, we established a ChIP-Seq assay in interphasic and mitotic ES populations obtained by nocodazole treatment and mitotic shake-off (Methods online). Binding profiles of Esrrb were produced in wildtype E14Tg2a and in transgenic EKOiE cells cultured with Dox. EKOiE cells depleted for Esrrb (-Dox for 48h) were used as negative controls. We found that Esrrb remains bound at a subset of its interphase targets during mitosis, suggesting that it acts as a bookmarking factor (Fig. 2a).

Using statistical and reproducibility parameters (Methods and Supplementary Fig. 3 online), we identified nearly 14.000 Esrrb binding regions in interphase and 1.980 in mitosis (Supplementary Table 1 online). Albeit at different levels (Supplementary Fig. 3 online), mitotic binding regions were also bound by Esrrb in interphase (thereafter, Bookmarked; Fig. 2b, c, d). Nine regions were used for validation by quantitative PCR and titration experiments, excluding that remnant interphase cells in our mitotic preparations (<5\%; Methods online) may affect the observed Esrrb binding profiles (Supplementary Fig. 4 online).

We observed that Esrrb binding levels in mitosis followed a bi-modal distribution (Fig. 2b), with 6.000 regions showing very low levels. At these regions Esrrb binding was never detected in mitosis (Supplementary Fig. 3 online), indicating that Esrrb is efficiently evicted (thereafter, Lost; Fig. 2e). The remaining 8.000 regions include the 1.980 regions identified as Bookmarked. Excluding those, the 
levels of mitotic Esrrb binding did not significantly differ from the negative controls and the detection of Esrrb failed in at least one of our four independent replicates (Supplementary Fig. 3 online). Even though a subset of these regions may be inefficiently bound in mitosis, we adopted a conservative approach and considered them as Lost for subsequent analyses.

We conclude that Esrrb remains robustly bound to $10-15 \%$ of its interphase targets (Bookmarked regions; Fig. 2c), and shows no or labile mitotic binding at other regions (Lost regions; Fig. 2c). Of note, $\sim 55 \%$ of all ES-specific super-enhancers ${ }^{31}$ such as those of key pluripotency factors (Sox2, Tbx3 and Klf4), together with other regulatory regions of important genes are mitotically bound by Esrrb (Supplementary Fig. 5 online).

\section{Relationships between mitotic Esrrb binding and chromatin status in interphase.}

In mitosis, Esrrb binding is generally reduced at Bookmarked regions compared to interphase (Fig. 2b, c). Moreover, Bookmarked regions show higher Esrrb binding in interphase than Lost regions (Fig. 2c). Using published datasets ${ }^{3,32,33}$, both Bookmarked -B- and Lost -L- regions could be divided into two clusters (Fig. 3a) according to their chromatin status in interphase: either particularly enriched for binding of other TFs and active histone marks (B1 and L1) or not (B2 and L2; Supplementary Fig. 6a online). However, direct quantitative comparisons showed that, whilst B2/L2 display homogeneously low levels (Supplementary Fig. 6a online), B1 regions exhibit higher enrichment for TFs, the transcriptional machinery and euchromatin-associated histone marks than L1 regions (box plots in Fig. 3a). For instance, both B1 promoters and enhancers display higher levels of Nanog, RNAPII and H3K27ac than their L1 counterparts losing Esrrb in mitosis (Fig. 3b). Other marks of activity followed an identical trend (Supplementary Fig. 6b online). Conversely, although it is generally detected at very low levels, the repressive $\mathrm{H} 3 \mathrm{~K} 27 \mathrm{me} 3$ mark is slightly more abundant in L1 than in B1 regions, particularly at promoters (Supplementary Fig. 6b online).

Gene ontology analysis (Fig. 4a) showed that genes associated with B1 regions tend to be expressed in undifferentiated cells of pre-implantation embryos. In contrast, genes associated with L1 
regions are expressed in non-pluripotent extra-embryonic tissues. Genes associated to B2/L2 tend to be expressed at later stages of development. In accordance, analysis of hallmarks of transcriptional activity in published genome-wide datasets ${ }^{33}, 34$, indicates that genes located in the vicinity of Bookmarked regions are more actively transcribed than those located nearby Lost regions (B1 versus L1 and B2 versus L2; Fig. 4b).

Notwithstanding that Esrrb binds regions of different chromatin constitution (B1/2 an L1/2), mitotic Esrrb binding marks active enhancers and promoters of genes highly transcribed in ES cells and developmentally associated with pre-implantation pluripotency.

\section{Mitotic Esrrb binding is driven by specific DNA binding to its motif.}

Using de-novo motif discovery algorithms we found that the canonical extended estrogen receptor half-site characteristic of Esrrb was strongly enriched at Bookmarked regions, with nearly $90 \%$ of these regions containing at least one motif (Fig. 5). Conversely, only $32 \%$ of Lost regions had Esrrb motifs, suggesting that specific DNA-protein interactions are required for mitotic binding of Esrrb. To test this, we mutated 3 residues of Esrrb that mediate the principal base-specific interactions with DNA (Methods online). This binding-defective mutant (Supplementary Fig. 7b online) did not decorate the mitotic chromosomes to the extent of wild-type Esrrb-GFP (Fig. 5b and Supplementary Fig. 7c online).

An undescribed longer Esrrb motif extended by 3 bases (AGT) was prominent in Bookmarked regions (63\%). In Lost regions, however, only $14 \%$ displayed a long motif and of lower quality (Fig. 5a and Supplementary Fig. 7a online). Hence, the long motif may play a specific role in Esrrb bookmarking. To test this, we used CRISPR to turn the single long Esrrb binding motif present under the major Esrrb peak at the Esrrb enhancer into a short version (Supplementary Fig. 7d online), with no further modification of the locus. In this mutant cells, we could not measure any significant difference in levels of Esrrb binding in interphase and mitosis (Supplementary Fig. 7e online).

To directly test whether long and short motifs are sufficient to drive mitotic Esrrb binding, we used recombinase mediated cassette exchange (RMCE) to introduce a tandem array of 4 long or short 
motifs in an identical genomic location (Methods online). We observed that Esrrb was efficiently recruited in interphase and its binding maintained during mitosis both for the long and short motifs

(Fig. 5c, d) and irrespectively of the locus of integration (Clones \#1 and \#2). While the significance of the 3 extra-bases that we have identified remains unclear, these experiments demonstrate that the presence of Esrrb binding sites is sufficient to drive its mitotic retention. In agreement, the presence of any Esrrb motif in Bookmarked regions is associated with increased binding in mitosis and more efficient mitotic retention, as revealed in our ChIP-Seq results (Supplementary Fig. 7f, g online).

We conclude that the interaction of Esrrb with its cognate binding site correlates with efficient mitotic binding at endogenous loci and is sufficient to drive the mitotic retention at ectopic sites. For the $10 \%$ of Bookmarked regions lacking Esrrb binding sites, however, other mechanisms must be invoked; for example protein-protein interactions with other factors. In line with this, Bookmarked regions lacking a motif display unexpectedly high enrichment levels of other pluripotency TFs in interphase (Supplementary Fig. 7f, h online).

\section{Mitotic Esrrb binding is dynamic.}

The retention of Esrrb on mitotic chromatin could either result from the permanence of Esrrb protein already loaded on chromatin at the end of G2, or from de novo and dynamic binding of Esrrb in mitosis. To discriminate between these two possibilities, we performed FRAP experiments (Fig. 6a), a strategy that has been widely used to infer TF binding dynamics ${ }^{35-37}$ : while a fast fluorescent recovery is associated with rapid ON/OFF binding transitions, a slow recovery results from a decreased turnover of photo-bleached and fluorescent molecules, indicating more stable interactions. In interphase, EsrrbGFP signal promptly recovered after bleaching (80\% recovery in 5s, Fig. 6a, b), indicating that Esrrb binding is dynamic. Strikingly, the specific spatial localisation of the GFP signal resulting from the decoration of mitotic chromosomes by Esrrb-GFP was also recovered after bleaching, and with faster dynamics than in interphase ( $80 \%$ in 2s, Fig. 6a, b). As a control, DNA binding Errb mutant showed even 
faster GFP recovery rates (Fig. 6b), confirming that the delay in recovery observed for wild-type Esrrb results from its DNA binding ability both in interphase and mitosis.

These results indicate that Esrrb is able to bind de novo to mitotic chromatin and that the observed retention likely results from continuous cycles of transient and fast interactions of Esrrb with DNA. Together with the previous analysis of Esrrb binding sites, this suggests that Esrrb behaves as a canonical TF in mitosis in terms of its binding to DNA.

\section{Mitotic Esrrb binding correlates with transcription activation in early-G1.}

To explore the functional consequences of mitotic Esrrb binding on gene expression we introduced a new cell-cycle reporter into Dox-inducible EKOiE cells (Methods online). This reporter system is based on the fusion of residues 1-156 of murine Cyclin A2 (Ccna2) to GFP, driving its mitotic degradation starting from prometaphase ${ }^{38,39}$. When used in conjunction with the live DNA stain Hoechst, this system allows sorting cells as they proceed through $\mathrm{G} 1, \mathrm{~S}$ and $\mathrm{G} 2$, without compromising viability (Supplementary Fig. 8a online). Ccna2-GFP cells were grown in the presence or absence of Dox (24h withdrawal), isolated by FACS in early-G1, late-G1 and G2 fractions (Fig. 7a), and analysed by RNASeq using a pipeline enabling to quantify both mRNA and pre-mRNA levels (Methods and Supplementary Table 2 online).

Overall, around 500 genes were up/downregulated by Esrrb at both mRNA and pre-mRNA levels (Fig. 7b and Supplementary Table 3 online). 168 and 206 genes showed prompt positive or negative response already in early-G1 and were coherently affected by the loss of Esrrb during all cellcycle phases (Supplementary Fig. 8b online). The number of Esrrb-responsive genes increased during cell-cycle progression (Fig. 7b), with around half showing changes only in G2. Early-G1 responsive genes associated with a Bookmarked region include pluripotency TFs (Tfcp2I1, Tbx3 and KIf4) and regulators of differentiation (Id2-3), chromatin (Dnmt3L) and embryonic cell-cell interactions (Jam2; Fig. 6c). Notably, Esrrb-up/downregulated genes in early-G1 are also up/downregulated in ground-state conditions of systematic self-renewal ${ }^{40}$ (Supplementary Fig. $8 c$ online). 
Almost $75 \%$ of genes activated by Esrrb in early-G1 were located within topological associated domains ${ }^{41}$ (TADs) containing at least one Esrrb bookmarked region (Fig. 7d). In contrast, only $55 \%$ of the genes regulated by Esrrb only in $\mathrm{G} 2$ were located within bookmarked TADs, as observed for an equivalent set of random genes. Similarly, nearly $25 \%$ of the genes upregulated by Esrrb in early-G1 were within $25 \mathrm{~kb}$ of a Bookmarked region. In contrast, genes downregulated by Esrrb tend to be further away from Bookmarked regions (Fig. 7d).

Overall, the integration of our ChIP-Seq and RNA-Seq results indicate that mitotic Esrrb binding transcriptionally activates a subset of key genes in early-G1, including pluripotency regulators that in turn may control additional genes during the cell-cycle. This strongly suggests that Esrrb behaves as a canonical bookmarking factor. 


\section{Discussion}

We report here that Esrrb, a major pluripotency regulator $^{8,9,42}$, decorates the mitotic chromosomes of ES cells, an ability not shared by Nanog. We further demonstrate that Esrrb is specifically enriched at a subset of its interphase targets. Recently, other sequence-specific TFs have been shown to bind mitotic chromatin, suggesting that the mitotic retention of TFs may have been generally overlooked. Several common observations can be identified among recent studies ${ }^{27-29,43}$. First of all, only a subset of TF binding regions are effectively bound in mitosis, albeit at lower levels than in interphase. Whether this is due to technical reasons or reflects a particular behaviour of mitotic binding is unclear, but our FRAP analyses suggest that mitotic Esrrb is hyper-dynamic and likely less amenable to chemical cross-linking and robust detection by ChIP-Seq. Secondly, regions bound in mitosis tend to exhibit particularly robust binding in interphase and contain canonical DNA binding sites (Fig. 8). Similarly to Gata1, FoxA1 and Hnf1, Esrrb bookmarking requires its DNA-binding domain to interact with the mitotic chromatin. Moreover, we demonstrate that the experimental introduction of ectopic recognition motifs is sufficient to drive Esrrb binding in interphase and in mitosis. Additional mechanisms of mitotic retention may however exist, as we identified around $10 \%$ of mitotically bound regions lacking clear motifs. These regions exhibit high enrichment for other pluripotency TFs, suggesting that additional regulators may bind mitotic chromatin in ES cells, a possibility that needs to be comprehensively assessed (Fig. 8). Thirdly, the mitotic retention of TFs is associated with widespread coating of the chromosomes, which is hardly explained by binding at a subset of enhancers and promoters. The mutation of FoxA1 DNA binding domain leads to the loss of binding at regions containing binding sites, but not to the loss of the chromosome-wide coating ${ }^{28}$. Conversely, mutations in the Hnf1 DNA-binding domain abolish chromatid coating but not site specific DNA binding in interphase ${ }^{43}$. In the case of Esrrb, we show that the mutation of its DNA-binding domain abolishes both binding at specific loci in interphase and the general coating of mitotic chromosomes. Hence, the decoration of the mitotic chromosomes is mediated by different types of molecular interactions 
depending on the TF under study. This differential behaviour of TFs needs to be directly investigated, as it calls into question the idea that mitotic chromosomes are intrinsically refractory to scanning by TFs. New understanding of the nature of mitotic chromatin needs to be attained should we want to uncover the exact nature and relevance of mitotic bookmarking.

We have observed that, in interphase, regions bound by Esrrb tend to be either highly enriched for signatures of active chromatin (B1/L1 regions) or not marked by any specific feature (B2/L2), a chromatin state reminiscent of naïve chromatin ${ }^{44,45}$. However, direct comparison of B1 and L1 regions clearly shows that mitotic Esrrb binding is associated with regions of particularly high activity in interphase. Most notably, H3K27ac and RNAPII enrichment show the highest fold difference between bookmarked and lost regions. That Esrrb interacts with several members of the basal transcription complex, coactivators and histone acetyl-transferases ${ }^{46}$ may provide a molecular understanding of the consequences of mitotic binding. Together with previous reports ${ }^{25,27-29,43}$, our findings indicate that mitotic binding of Esrrb and of other factors, enables the maintenance of active chromatin properties at key regulatory regions that, in turn, activate important genes during interphase. Esrrb binding in mitosis seems to result in the rapid upregulation of important pro-self-renewal genes (KIf4, Tfcp2I1 and $T b \times 3$ ) as ES cells re-enter into interphase (Fig. 8). The fact that alterations in both transcriptional output and mature mRNA accumulation are evident already in early-G1 argues in favour of an almost immediate resumption of transcription. Therefore, Esrrb should be considered as a mitotic bookmarking factor ${ }^{4-49}$ contributing to the long term maintenance of pluripotency by jumpstarting the post-mitotic resuscitation of the pluripotency network and counterbalancing the inherent propensity of ES cells to undergo differentiation from $\mathrm{G} 1^{15,50,51}$.

As shown for Gata1 ${ }^{29}$, taking into consideration only mitotic binding allows a contraction of the gene ontology associations of Esrrb binding regions to particularly relevant genes, those highly expressed during pre-implantation development when pluripotency is established. Hence, the observation that in early embryos mitotic Esrrb binding is operational opens the possibility of a direct involvement of this process when Esrrb expression becomes restricted to the pluripotent $\mathrm{ICM}^{30,52}$. 
Similarly, the generation of induced pluripotent stem (iPS) cells has been shown to transit from a stochastic to a more deterministic process coinciding with the reactivation of endogenous Esrrb expression $^{53}$. Thus, naïve pluripotency may only be attained when the regulatory network becomes mitotically self-sustainable through Esrrb binding.

Whilst ES cells self-renew indefinitely, in the embryo pluripotency is a transient state that is rapidly extinguished upon differentiation. We speculate that Esrrb bookmarking might be a key phenomenon enabling the transient epigenetic maintenance of pluripotency in the early embryo without the need to establish mitotically heritable chromatin marks which, although reversible, impose further molecular constrains to the timely extinction of pluripotency. More generally, we believe that mitotic bookmarking by TF has enormous advantages over other systems of mitotic inheritance in transiently expanded cell types such as activated stem cells and progenitors. 


\section{End notes}

Acknowledgements: We are grateful to lan Chambers for allowing N.F and P.N to pursue observations made initially in his laboratory on the chromosomal retention of Esrrb during mitosis. We are grateful to A. Rada-Iglesias, G. Cecere, S. Tajbakhsh, M. Yaniv and members of our laboratory for critical reading of the manuscript. We acknowledge B. Jagla for running the iRNA-seq pipeline and P. Clerc for assistance with fluorescence microscopy. P.N acknowledges financial support to the Institut Pasteur, CNRS and the ANR Laboratoire d'Excellence Revive (Investissement d'Avenir; ANR-10-LABX-73). N.F was funded by an EMBO Long Term Fellowship (ALTF 876-2013) and a Marie-Curie Intra-European Fellowship (EFIMB - 626705).

Author Contribution: This study was conceived by N.F and P.N. All experiments were carried out by N.F with the exception of embryo work performed by S.V.P, S.B and M.C.T. In addition, A.D, E.G.T and A.M provided technical help and F.M contributed to the analysis of FRAP assays. C.P performed next generation sequencing and P.N conducted the Bioinformatic analyses. All experiments were analysed and interpreted by N.F and P.N. The manuscript was written by P.N with inputs from N.F.

Competing Financial Interests: The authors declare that they have no financial competing interests. 


\section{Figure Legends:}

Figure 1: Esrrb decorates the mitotic chromosomes. (a) Representative images of Esrrb-GFP (top) or Nanog-GFP (bottom) fusion proteins in live cells cultured with Hoechst. (b) Esrrb immuno-fluorescence (green in the merge) on a metaphasic chromosome counterstained with DAPI (blue in the merge). (c) Representative images of live cells cultured with Hoechst and expressing Esrrb-tdTomato from both Esrrb endogenous alleles. (d) Esrrb-tdTomato and GFP mRNAs were simultaneously micro-injected in a single blastomere of a 2-cell embryo; at the compacted morula stage GFP-positive cells stained with Hoechst exhibited clear mitotic retention of Esrrb-tdTomato (arrows). Scale bars represent 10 $\mu \mathrm{m}$ for ES cells and embryos; $1 \mu \mathrm{m}$ for single chromosomes.

Figure 2: Esrrb binds a subset of its interphase targets in mitosis. (a) Illustrative binding profile of Esrrb across the Sox 2 extended locus (Controls are EKOiE cells cultured without Dox for $48 \mathrm{~h}$ to deplete Esrrb). (b) Histogram showing the distribution of Esrrb binding levels at all regions in interphase (Int. blue line) and mitosis (Mit. red line). The red-coloured area of the mitosis-line corresponds to the Bookmarked regions; the white area to the Lost regions. (c) Average Esrrb binding profiles at Bookmarked and Lost regions ( $\mathrm{Y}$-axis represents coverage in base per kb per million). (d, e) Examples of regions (6.7kb) retaining Esrrb in mitosis (d) or losing its binding (e). The closest gene to each peak is indicated.

Figure 3: Mitotic Esrrb binding at regions of high activity in interphase. (a) Hierarchical clustering of Esrrb binding regions (numbers on the left) based on enrichment for the indicated marks or factors ${ }^{3}$ 32, 33. The corresponding boxplots (median, $25^{\text {th }} / 75^{\text {th }}$ percentiles) and respective whiskers (median plus/minus the interquartile range multiplied by 2) are shown below the heatmap for B1 (red) and L1 (grey) regions. The p-value of the difference between B1 and L1 for each factor is indicated (Kruskal- 
Wallis ANOVA followed by Dunn's multiple comparison test). (b) Average binding profile of Nanog, Ser5-phosphorylated RNAPII and H3K27ac ${ }^{3,32,33}$ at B1 (red) versus L1 (black) promoters and enhancers.

Figure 4: Mitotic Esrrb binding occurs in the vicinity of key, highly transcribed genes, in vivo and in vitro. (a) Major embryonic sites and stages of expression of genes associated with the clusters identified in Figure $3 a$ are indicated alongside the $-\log 10$ binomial p-value. (b) Boxplot (same parameters as in Figure 3a) showing the expression levels (RPKM) measured by nuclear RNA-Seq ${ }^{34}$ (an accurate proxy for transcriptional pre-mRNA outputs) and ChIP-Seq enrichment (RPKM) of transcriptional elongation hallmarks (RNAPII-S2 and $\mathrm{H} 3 \mathrm{~K} 36 \mathrm{me} 3^{33}$ ) across transcription units located within $25 \mathrm{~kb}$ of B1, B2, L1 and L2 regions. The $p$-values were calculated as in Figure 3 (B1 n=2013 ; B2 $n=6008 ; L 1 \quad n=6008 ; L 2 n=1156)$.

Figure 5: The Esrrb DNA binding motif drives mitotic binding. (a) The Esrrb consensus is shown together with the main motifs discovered in Lost and Bookmarked regions. The histogram shows the fraction of Bookmarked and Lost regions containing long (red) or only short (blue) Esrrb motifs. (b) Representative images of mutant (mut) Esrrb-GFP fusions in live cells cultured with Hoechst, imaged in parallel to that shown in Fig. 1a. Scale bars represent 10 $\mu \mathrm{m}$. (c) Average Esrrb ChIP results shown as percentage of immunoprecipitation ( $n=4$; error bars represent sd) at a Bookmarked (Pdgfa) and a Lost (Twistnb) region (see Fig. 1d, e), and at synthetic binding sites constituted of 4 tandem repetitions of short (upper panel) or long (lower panel) motifs. 'Non binding' represents the average of two genomic positions not bound by Esrrb (Actin promoter and a region 3' to the Esrrb gene). In each cell line (clone $\# 1$ and \#2), the short and long synthetic binding sites were introduced at identical genomic positions. (d) Percentage of Esrrb mitotic retention calculated from (c) for the long and short synthetic binding sites in 2 independent clones. 
Figure 6: Mitotic Esrrb binding is dynamic. (a) Esrrb-GFP signal before and after bleaching, at the indicated time (seconds), in interphase (up) and on mitotic chromosomes (down). Scale bars represent 10 $\mathrm{m}$. (b) Quantifications of FRAP experiments in interphase (left) and mitosis (right) using wild-type (WT; black) or mutant-Esrrb-GFP (Mut; green) fusion proteins (WT interphase $n=13$; WT mitosis $n=14$; Mut interphase $n=12$; Mut mitosis $n=13$ ). The $Y$-axis shows the mean percentage of fluorescence; the $\mathrm{X}$-axis the number of seconds after bleaching. The highlighted values represent the time required to recover $80 \%$ of the starting fluorescence signal.

Figure 7: Esrrb transcriptional control along the cell-cycle. (a) FACS profile of EKOiE cells expressing Ccna2-GFP and cultured with Hoechst. The sorted populations used for RNA-Seq are highlighted. (b) Number of Esrrb-responsive genes in early-G1, late-G1 and G2. (c) Average fold change in expression of selected pre-mRNA in early-G1 (RNA-Seq, white, versus RT-qPCR, black $-n=6$ for all pre-mRNA analysed except for $\operatorname{Tbx3}(n=3)$ and Suz12 $(n=4)$; error bars represent s.e.m). nd denotes that Id3 premRNA is not amenable for detection by RNA-Seq. (d) Percentage of Esrrb-responsive genes at each cell-cycle phase laying within a bookmarked TAD or near a bookmarked peak. In grey, average and s.d for 20 randomly selected sets of 200 genes. The p-values were calculated with a Fisher's exact test.

Figure 8: Potential Esrrb function as a mitotic bookmarking factor. This schematic diagram highlights that Esrrb mitotically binds at a subset of its interphase targets; in particular those having canonical Esrrb DNA binding motifs. Additional unknown factors may participate into the mitotic retention of Esrrb binding. Binding of Esrrb during mitosis takes place at promoters and enhancers associated with high transcriptional activity in undifferentiated cells and is enriched in the vicinity of genes responding to Esrrb in early G1. 


\section{References}

1. Smith, A.G. et al. Inhibition of pluripotential embryonic stem cell differentiation by purified polypeptides. Nature 336, 688-690 (1988).

2. Kim, J., Chu, J., Shen, X., Wang, J. \& Orkin, S.H. An extended transcriptional network for pluripotency of embryonic stem cells. Cell 132, 1049-1061 (2008).

3. Chen, X. et al. Integration of external signaling pathways with the core transcriptional network in embryonic stem cells. Cell 133, 1106-1117 (2008).

4. Chambers, I. et al. Nanog safeguards pluripotency and mediates germline development. Nature 450, 1230-1234 (2007).

5. Nichols, J. et al. Formation of pluripotent stem cells in the mammalian embryo depends on the POU transcription factor Oct4. Cell 95, 379-391 (1998).

6. Niwa, H., Miyazaki, J. \& Smith, A.G. Quantitative expression of Oct-3/4 defines differentiation, dedifferentiation or self-renewal of ES cells. Nature genetics 24, 372-376 (2000).

7. Masui, S. et al. Pluripotency governed by Sox2 via regulation of Oct3/4 expression in mouse embryonic stem cells. Nat Cell Biol 9, 625-635 (2007).

8. Festuccia, N. et al. Esrrb is a direct Nanog target gene that can substitute for Nanog function in pluripotent cells. Cell stem cell 11, 477-490 (2012).

9. Martello, G. et al. Esrrb is a pivotal target of the gsk3/tcf3 axis regulating embryonic stem cell self-renewal. Cell Stem Cell 11, 491-504 (2012).

10. Kopp, J.L., Ormsbee, B.D., Desler, M. \& Rizzino, A. Small increases in the level of Sox2 trigger the differentiation of mouse embryonic stem cells. Stem Cells 26, 903-911 (2008).

11. Chambers, l. et al. Functional expression cloning of Nanog, a pluripotency sustaining factor in embryonic stem cells. Cell 113, 643-655 (2003).

12. Stead, E. et al. Pluripotent cell division cycles are driven by ectopic Cdk2, cyclin A/E and E2F activities. Oncogene 21, 8320-8333 (2002).

13. White, J. et al. Developmental activation of the Rb-E2F pathway and establishment of cell cycle-regulated cyclin-dependent kinase activity during embryonic stem cell differentiation. Mol Biol Cell 16, 2018-2027 (2005).

14. Faast, R. et al. Cdk6-cyclin D3 activity in murine ES cells is resistant to inhibition by p16(INK4a). Oncogene 23, 491-502 (2004).

15. Dalton, S. Linking the Cell Cycle to Cell Fate Decisions. Trends Cell Biol 25, 592-600 (2015).

16. Martinez-Balbas, M.A., Dey, A., Rabindran, S.K., Ozato, K. \& Wu, C. Displacement of sequence-specific transcription factors from mitotic chromatin. Cell 83, 29-38 (1995).

17. Tsumura, A. et al. Maintenance of self-renewal ability of mouse embryonic stem cells in the absence of DNA methyltransferases Dnmt1, Dnmt3a and Dnmt3b. Genes Cells 11, 805-814 (2006).

18. Leeb, M. et al. Polycomb complexes act redundantly to repress genomic repeats and genes. Genes Dev 24, 265-276 (2010).

19. Lehnertz, B. et al. Suv39h-mediated histone H3 lysine 9 methylation directs DNA methylation to major satellite repeats at pericentric heterochromatin. Curr Biol 13, 1192-1200 (2003).

20. Tachibana, M. et al. G9a histone methyltransferase plays a dominant role in euchromatic histone H3 lysine 9 methylation and is essential for early embryogenesis. Genes Dev 16, 1779-1791 (2002).

21. De Los Angeles, A. et al. Hallmarks of pluripotency. Nature 525, 469-478 (2015).

22. Michelotti, E.F., Sanford, S. \& Levens, D. Marking of active genes on mitotic chromosomes. Nature 388, 895-899 (1997).

23. Xing, H., Vanderford, N.L. \& Sarge, K.D. The TBP-PP2A mitotic complex bookmarks genes by preventing condensin action. Nature cell biology 10, 1318-1323 (2008). 
24. Zaidi, S.K. et al. Mitotic partitioning and selective reorganization of tissue-specific transcription factors in progeny cells. Proceedings of the National Academy of Sciences of the United States of America 100, 14852-14857 (2003).

25. Blobel, G.A. et al. A reconfigured pattern of MLL occupancy within mitotic chromatin promotes rapid transcriptional reactivation following mitotic exit. Molecular cell 36, 970-983 (2009).

26. Young, D.W. et al. Mitotic occupancy and lineage-specific transcriptional control of rRNA genes by Runx2. Nature 445, 442-446 (2007).

27. Verdeguer, F. et al. A mitotic transcriptional switch in polycystic kidney disease. Nat Med 16, 106-110 (2009).

28. Caravaca, J.M. et al. Bookmarking by specific and nonspecific binding of FoxA1 pioneer factor to mitotic chromosomes. Genes \& development 27, 251-260 (2013).

29. Kadauke, S. et al. Tissue-specific mitotic bookmarking by hematopoietic transcription factor GATA1. Cell 150, 725-737 (2012).

30. Guo, G. et al. Resolution of cell fate decisions revealed by single-cell gene expression analysis from zygote to blastocyst. Dev Cell 18, 675-685 (2010).

31. Whyte, W.A. et al. Master transcription factors and mediator establish super-enhancers at key cell identity genes. Cell 153, 307-319 (2013).

32. Shen, Y. et al. A map of the cis-regulatory sequences in the mouse genome. Nature 488, 116120 (2012).

33. Brookes, E. et al. Polycomb associates genome-wide with a specific RNA polymerase II variant, and regulates metabolic genes in ESCs. Cell Stem Cell 10, 157-170 (2012).

34. Bulut-Karslioglu, A. et al. Suv39h-dependent H3K9me3 marks intact retrotransposons and silences LINE elements in mouse embryonic stem cells. Mol Cell 55, 277-290 (2014).

35. Farla, P. et al. The androgen receptor ligand-binding domain stabilizes DNA binding in living cells. J Struct Biol 147, 50-61 (2004).

36. Sauer, M. et al. C-terminal diversity within the p53 family accounts for differences in DNA binding and transcriptional activity. Nucleic Acids Res 36, 1900-1912 (2008).

37. Mueller, F., Stasevich, T.J., Mazza, D. \& McNally, J.G. Quantifying transcription factor kinetics: at work or at play? Crit Rev Biochem Mol Biol 48, 492-514 (2013).

38. den Elzen, N. \& Pines, J. Cyclin A is destroyed in prometaphase and can delay chromosome alignment and anaphase. J Cell Biol 153, 121-136 (2001).

39. Geley, S. et al. Anaphase-promoting complex/cyclosome-dependent proteolysis of human cyclin A starts at the beginning of mitosis and is not subject to the spindle assembly checkpoint. J Cell Biol 153, 137-148 (2001).

40. Marks, H. et al. The transcriptional and epigenomic foundations of ground state pluripotency. Cell 149, 590-604 (2012).

41. Dixon, J.R. et al. Topological domains in mammalian genomes identified by analysis of chromatin interactions. Nature 485, 376-380 (2012).

42. Feng, B. et al. Reprogramming of fibroblasts into induced pluripotent stem cells with orphan nuclear receptor Esrrb. Nat Cell Biol 11, 197-203 (2009).

43. Lerner, J. et al. Human mutations affect the epigenetic/bookmarking function of HNF1B. Nucleic Acids Res (2016).

44. Soufi, A., Donahue, G. \& Zaret, K.S. Facilitators and impediments of the pluripotency reprogramming factors' initial engagement with the genome. Cell 151, 994-1004 (2012).

45. Soufi, A. et al. Pioneer transcription factors target partial DNA motifs on nucleosomes to initiate reprogramming. Cell 161, 555-568 (2015).

46. van den Berg, D.L. et al. An Oct4-centered protein interaction network in embryonic stem cells. Cell Stem Cell 6, 369-381 (2010).

47. Zaidi, S.K. et al. Mitotic bookmarking of genes: a novel dimension to epigenetic control. Nat Rev Genet 11, 583-589 (2010). 
48. Kadauke, S. \& Blobel, G.A. Mitotic bookmarking by transcription factors. Epigenetics \& chromatin 6, 6 (2013).

49. Zaret, K.S. Genome reactivation after the silence in mitosis: recapitulating mechanisms of development? Dev Cell 29, 132-134 (2014).

50. Sela, Y., Molotski, N., Golan, S., Itskovitz-Eldor, J. \& Soen, Y. Human embryonic stem cells exhibit increased propensity to differentiate during the $\mathrm{G} 1$ phase prior to phosphorylation of retinoblastoma protein. Stem Cells 30, 1097-1108 (2012).

51. Pauklin, S. \& Vallier, L. The cell-cycle state of stem cells determines cell fate propensity. Cell 155, 135-147 (2013).

52. Boroviak, T., Loos, R., Bertone, P., Smith, A. \& Nichols, J. The ability of inner-cell-mass cells to self-renew as embryonic stem cells is acquired following epiblast specification. Nature cell biology 16, 516-528 (2014).

53. Buganim, Y. et al. Single-Cell Expression Analyses during Cellular Reprogramming Reveal an Early Stochastic and a Late Hierarchic Phase. Cell 150, 1209-1222 (2012). 


\section{Supplementary Figures Legends}

Supplementary Figure 1: Cell lines used in this study. (a) Esrrb immuno-fluorescence on EKOiE cells cultured in the presence or absence of Dox ( $24 \mathrm{~h}$ withdrawal). Scale bars represent $30 \mu \mathrm{m}$ (b) The selfrenewal efficiency of wild-type E14Tg2a, TNG, EKOiE and the respective derivative cell lines was assessed by Alkaline Phosphatase staining of cell colonies obtained 6 days after clonal plating in the indicated conditions. The graph shows the average and s.d of two independent experiments, each performed in duplicate wells. E14Tg2a cells self-renew exclusively in the presence of LIF. Upon introduction of a Nanog-GFP transgene, self-renewal efficiency increases in the presence of LIF and is rescued in its absence. As expected, TNG cells (Nanog heterozygous) exhibit reduced self-renewal as compared to E14Tg2a $a^{4}$. Upon knock-in of TdTomato at both Esrrb alleles, so that only Esrrb-TdT fusion protein is expressed in the cells, self-renewal efficiency is unaffected. EKOiE cells cultured without Dox show extremely severe defects in self-renewal efficiency as compared to Dox-treated cells, demonstrating the importance of Esrrb. In the presence of Dox, EKOiE cells also exhibit LIFindependent self-renewal. Upon the introduction of a constitutive Esrrb-GFP transgene, EKOiE cells are able to self-renew both in presence or absence of Dox; they also exhibit LIF-independent self-renewal. We conclude from this analysis that all the GFP and TdT fusion proteins (Nanog and Esrrb) are functional.

Supplementary Figure 2: Validation of Esrrb retention on mitotic chromosomes. (a) Representative live-imaging of nocodazole-treated EKOiE cells carrying an Esrrb-GFP transgene and cultured in the absence of Dox. DNA was counterstained with Hoechst. Note that all mitotic cells display chromosomal retention of Esrrb. (b) Representative live-imaging of E14Tg2a cells expressing a Nanog-GFP transgene. DNA was counterstained with Hoechst. All mitotic cells display exclusion of Nanog from the chromosomes. (c) Representative live-imaging of nocodazole-treated Esrrb-TdTomato cells. DNA was counterstained with Hoechst. All mitotic cells show enrichment of Esrrb on the chromosomes. (d) 
Representative Esrrb immuno-staining on a full karyotype from EKOiE cells cultured in the presence (top) or absence (bottom) of Dox. DNA was counterstained with DAPI. (e) SNAP-Cell TMR-Star staining on a karyotype from EKOiE cells constitutively expressing Snap-tagged Esrrb and cultured without Dox. (f) Simultaneous immuno-staining for Nanog and Esrrb on a karyotype obtained from wild-type E14Tg2a cells. Scale bars represent $10 \mu \mathrm{m}$.

Supplementary Figure 3: Additional information on Esrrb binding regions. (a) The table highlights the criteria used to categorise peaks as bookmarked or lost. Classes_1,2,3 were considered as bookmarked (see Methods for further details). ${ }^{*}$ Note that in class_1 all peaks (with one exception) were detected in at least one interphase dataset; they cannot be considered as mitotic-specific. (b) Esrrb average binding profile at the 5 classes of regions shown in (a) in both Interphase (Int.) and Mitosis (Mit.). All 5 classes show clear enrichment in interphase. Note that in mitosis Class_5 exhibits a complete depletion of Esrrb, whilst Class_4 shows low levels of Esrrb binding. The Y-axis represents alignment coverage expressed in bases per kb per million. (c) Aligned heat map of Esrrb density across the 5 classes of Esrrb binding regions. Note the focal accumulation of Esrrb at the centre of regions from all 5 classes in interphase (blue). In contrast, only Classes_1,2,3 show clear Esrrb binding in mitosis. (d) Heat-map of Esrrb enrichment levels (Log2_RPKM) at all binding regions in interphase (I) and mitosis (M) in all our datasets (E: Esrrb ChIP-Seq; C: Control obtained from Esrrb ChIP-Seq in EKOiE cultured without Dox; Mean represents the average of all shown replicates; Pooled represents enrichment levels after pooling together reads derived from all the replicates; Chen shows the enrichment levels in an independent ChIP-Seq ${ }^{3}$ for Esrrb in interphase (E: Esrrb ChIP-Seq; C: negative ChIP control against GFP). Note that across all regions the levels of Esrrb enrichment are significantly higher in interphase than in mitosis and all the controls. Also, note that in mitosis only Classes_1,2,3 show consistently high levels of enrichment. (e) Scatter plot representing enrichment levels in interphase ( $\mathrm{X}$-axis) versus mitosis ( $\mathrm{Y}$ axis) for the 5 classes of Esrrb binding regions. Note that Classes_1, 2 and 3 cluster together whilst Class_4 localises between Classes_1, 2 and 3 and Class_5. These analyses strongly support grouping 
together Classes_1,2,3 as Bookmarked and Classes_4,5 as Lost, even though a subset of Class_4 regions may display low levels of mitotic binding.

Supplementary Figure 4: ChIP-Seq validation by qPCR. (a) Six Bookmarked positions selected for spanning different levels of mitotic binding were selected along with five Lost positions and analysed by ChIP-qPCR for Esrrb binding ( $n=3$; error bars represent s.d) in interphase (blue) and mitosis (red) in EKOiE cells cultured with Dox. Interphasic EKOiE cells cultured in the absence of Dox were used to control for the specificity of our assay (dark grey). Moreover, 5 and $10 \%$ of chromatin prepared from Dox-treated EKOiE cells was spiked into -Dox chromatin to test whether the contaminant interphasic cells $(<5 \%)$ in our mitotic preparations (see Methods online) are sufficient to produce a level of enrichment similar to that observed in mitosis. At bookmarked positions we observe mitotic enrichment levels (percentage IP, Y-axis) significantly higher than at Lost positions. Moreover, even after introducing $10 \%$ of contaminant chromatin to EKOiE -Dox controls, the observed \%IP remains much lower than in mitotic cells for all Bookmarked positions tested. (b) Binding profile at three loci belonging to Class_1 (putative mitotic-specific peaks, see Supplementary Figure 3) illustrating that even though the peak detected in interphase is not statistically significant, it is of similar magnitude to that observed in mitotic cells. (c) ChIP-qPCR validation of interphasic and mitotic binding at the 3 loci shown in (b), presented as in (a).

Supplementary Figure 5: Examples of bookmarked genes. Bookmarked regions include $55 \%$ of ESspecific super-enhancers (e.g. Tbx3 and KIf4 or Sox2 in Figure 2a). Other noticeable regions bookmarked by Esrrb correspond to developmental genes (Otx2), chromatin remodelers (Ino80), Polycomb Group proteins (Jarid2), regulators of DNA methylation (Tet1), signalling mediators (Lif receptor) and metabolic genes (Eno1). Mitosis and interphase are shown at the same scale. 
Supplementary Figure 6: B1 regions are more active than L1 regions. (a) Enrichment levels of the indicated factors and chromatin modifications at the 4 clusters identified in Figure 3a. The upper and lower extremities of each shaded area are the 25th and 75th percentile of each dataset across the 4 clusters. (b) Average profiles of the indicated factors and marks across promoters and enhancers belonging to B1 and L1 clusters as shown in Fig. 3.

Supplementary Figure 7: Additional information on the relationships between Esrrb bookmarking and the presence of Esrrb binding motifs. (a) The best motifs discovered are shown together with the percentage of regions containing at least one. Note that the regions containing a long motif may also contain a short motif. In contrast, the percentages given for the regions contain a short motif do not contain long motifs. (b) ChIP-qPCR of wild-type (WT) and Mutant (Mut) Esrrb at several genomic positions (mean+/-s.e.m; $n=2$ ). The two variants are expressed in EKOiE or EKOiE Mut cells under the control of Dox (see methods; cells growing without Dox and not expressing Esrrb are used as a negative control - CtI). (c) Representative live-imaging of mutant Esrrb-GFP carrying three mutations in residues known to establish specific contacts with the bases of the motif. DNA is counterstained with Hoechst. Note that no mitotic cell displays focal accumulation on the chromosomes. Scale bars represent $10 \mu \mathrm{m}$. (d) ChIP-Seq profile of Esrrb in interphase and mitosis across the Esrrb locus (coverage represents bases per kb per million). The arrow and dashed box indicate the position of the major enhancer of Esrrb shown at higher resolution on the right. The sequence of the long motif identified at this peak is shown; the 3 bases mutated to CCC are underlined. (e) Esrrb ChIP-qPCR at the Esrrb enhancer expressed as percentage of immunoprecipitation (for E14Tg2a $n=7$ for interphase and 3 for mitosis; for EEt -edited to short- $\mathrm{n}=17$ for interphase and 8 for mitosis; error bars represent s.d) in E14Tg2a and in cells where the long motif was edited into a short motif (AGTTCAAGGTCA -> CCCTCAAGGTCA). No alteration of Esrrb binding in interphase or mitosis is observed (unpaired t-test). (f) Global quantification (RPKM) of Esrrb enrichment at Bookmarked regions depending on whether they contain a long motif (red), only a short motif (blue), or no Esrrb motif at all (grey). Note that regions with short or long motifs display 
significantly higher enrichment in interphase and lower in mitosis. (g) Calculation of the percentage of mitotic retention at the three groups of Esrrb binding regions shown in (f). The presence of a long or a short binding site is associated with increased bookmarking efficiency. (h) Quantification of enrichment levels (RPKM) of several pluripotency $\mathrm{TFs}^{3}$ in the regions described in (f). All factors are statistically over-enriched at Esrrb binding regions lacking Esrrb motifs. The p-value of the difference between regions with long, only short or no motifs is indicated (Kruskal-Wallis ANOVA followed by Dunn's multiple comparison test).

\section{Supplementary Figure 8: Additional information of the Ccna-GFP reporter system and Esrrb-} responsive genes. (a) A transgene expressing GFP fused to the domain of Cyclin A2 (Ccna) responsible for its mitotic degradation ${ }^{38,39}$ was introduced in EKOiE cells that were stained with Hoechst to quantify DNA content. Ccna-GFP shows low fluorescence in M and early G1 and accumulates gradually before the cells enter into S phase. Asynchronous cells (before sorting) are presented along with sorted Early-G1, Late-G1 and G2 cells replated and cultured for additional 8 hours. Approximate sorting gates are shown along with the percentages of cells falling in each gate to illustrate how EKOiE Ccna-GFP cells are able to resume cell cycle progression after sorting: the majority of cells sorted in early-G1 progressed to the beginning of the S phase; cells sorted in late-G1 largely entered $\mathrm{S}$ and many reached G2; cells in G2 were delayed but could enter mitosis. (b) Relative expression (Log2) of early-G1 responsive genes (Early-G1 cultured without Dox were set to 0 ) along the cell cycle. (c) Boxplot showing the log2 fold change of Esrrb-responsive early-G1 genes in cells cultured in ground state conditions (2i) versus serum-containing medium ${ }^{40}$. The distribution of the fold changes followed a normal distribution (D'Agostino \& Pearson normality test); therefore the p-values were calculated with a one-way Student test against a theoretical fold change of 0 . 
Supplementary Movie 1: Time-lapse of Esrrb-GFP cells. The movie shows Esrrb-GFP cells and illustrates the continuous association of Esrrb with the mitotic chromosomes as cells enter, progress and exit mitosis.

Supplementary Information Table 1: Esrrb enrichment at its binding regions. In this table the coordinates of all annotated peaks can be found along with the RPKM values calculated in each independent replicate.

Supplementary Information Table 2: Genome-wide expression analysis of EKOiE cells in early G1, late $\mathbf{G 1}$ and $\mathbf{G 2}$. In this file the results of the RNA-Seq conducted for this study are presented in 3 tables (exonic counts, intronic counts, gene expression changes as established using edgeR within the iRNAseq pipeline).

Supplementary Information Table 3: Esrrb-dependent genes in early G1, late G1 and G2. In this file 6 tables describing the differentially expressed genes identified at each analysed cell-cycle phase are presented along with their associated FC and FDR.

Supplementary Information Table 4: Primer sequences. Sequences of all primer pairs used to validate the ChIP-Seq and RNA-Seq are provided.

Supplementary Information Table 5: Global statistics of NGS analyses. In this file we provide global statistic parameters of our ChIP-Seq ( 2 tables) and RNA-Seq results ( 2 tables). 


\section{Methods:}

\section{Cell lines:}

No cell lines used in this study were found in the database of commonly misidentified cell lines that is maintained by ICLAC and NCBI Biosample. All the cell lines generated for this study were derived from E14Tg2a, tested for mycoplasma and their identity authenticated by several means including resistance to drug selection, PCR and sequencing of specific genomic regions.

EKOiE and EKOiE Mut: The first exon of both Esrrb alleles of E14Tg2a cells was flanked by LoxP sites as described $^{8}$ and transfected with a CAG-rtTA-IRES-blasticidin ${ }^{R}$ and a doxycycline inducible tdTomato transgenes flanked by heterotypic FRT sites. Flp recombinase mediated cassette exchange (RMCE) was then used to exchange tdTomato with an Esrrb-IRES-Puromycin ${ }^{R}$ cassette (wildtype or mutant Esrrb, see below). Both Esrrb alleles were then deleted by Cre recombinase.

EKOiE derivatives: EKOiE cells were stably transfected with CAG promoter driven wild-type Esrrb-5xGly-

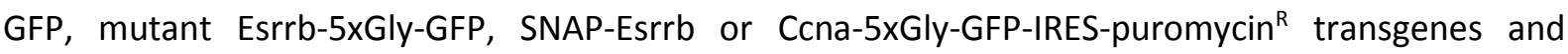
homogeneously expressing clones selected. In mutant Esrrb, Glu121, Lys124, and Lys128 are mutated to glycine ${ }^{54}$. Ccna-GFP includes only the mitotic degron of murine Cyclin A2 (residues 1-156) ${ }^{38,39}$.

Nanog-GFP: E14Tg2a cells were stably transfected with a CAG-Nanog-GFP-IRES-puromycin ${ }^{R}$ transgene.

EtdT: tdTomato-IRES-Blasticidin ${ }^{R}$ and tdTomato-IRES-Hygromycin ${ }^{R}$ were knocked-in downstream of the Esrrb ORF at each allele of TNG cells ${ }^{4}$.

ES cells carrying ectopic Esrrb binding sites: Two E14Tg2a clones bearing an FRT-flanked doxycyclineinducible tdTomato transgene and showing high and homogeneous expression were selected. 
tdTomato was exchanged for a $4 x$ binding site-IRES-Puromycin ${ }^{R}$ cassette by RMCE (long or short motifs). The $4 \mathrm{x}$ binding sites, derived from the Esrrb enhancer (AGAAGGAGCAACAGTTCAAGGTCATGCTCAGCTATA; AGT was mutated to CCC to generate a short motif), follow one another in the same orientation and spaced apart 30bp. Multi-copy integration of the binding site arrays was excluded by genomic DNA qPCR. Clones were cultured in doxycycline to avoid transgene heterochromatinisation.

Esrrb enhancer targeting (EEt) ES cells: E14Tg2a cells carrying a Ccna-GFP transgene were lipofected with a ssDNA oligo corresponding to $\mathrm{mm} 9$ chr12:87842618-87842736 with 3 bases of the Esrrb motif mutated (TGACCTTGAGGG), pGL3-U6-sgRNA-PGK-Puromycin vector (Addgene \#51133) driving expression of the gRNA ACAGAAGGAGCAACAGTTCA (NGG) and the CAS9/GFP expression vector (Addgene \#44719). 24h later GFP' cells were sorted (FacsARIA III; Becton-Dickinson) and single clones screened by genomic DNA qPCR using a primers specific for the WT or mutated allele. 400bp centred on the motif were sequenced and 3 clones carrying the desired substitution at both alleles selected. We excluded deletion of the genomic region extending beyond the PCR-screened region by qPCR. All lines carry homozygous mutations.

\section{Regular culture conditions:}

ES cells were cultured on 0.1\% gelatine (SIGMA, G1890-100G) in DMEM+GlutaMax-I (Gibco, 31966-

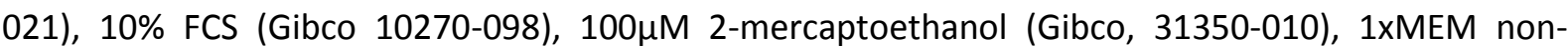
essential amino-acids (Gibco, 1140-035) and 10ng/ml recombinant LIF (MILTENYI BIOTEC, 130-099895). Cells were passaged 1:10 every 2-3 days. Clonal assays were performed by plating 600 cells per well of a 6 wells plate and after six days an Alkaline Phosphatase kit (Sigma, 86R-1KT) was used to identify undifferentiated colonies using a stereomicroscope (NIKON-SMZ1500). 


\section{Microinjection:}

All experiments were conducted according to the French and European regulations (EC Directive 86/609, French Law 2001-486) and were approved by the Institut Pasteur ethics committee $\left(\mathrm{n}^{\circ} 2012-\right.$ 0011). (C57BL/6xSJL/J) F1 female mice were purchased from Janvier and mated with CD1-IGS male mice purchased from Charles River Laboratories, France. Fertilized eggs were obtained from 6 superovulated 3 weeks old females submitted to intra-peritoneal injection of Chronogest PMSG (2.5units/mouse, Centravet) followed by Chorulon (5units/mouse, Intervet) $42-48 \mathrm{~h}$ later and then mated overnight with 6 males. Single blastomere microinjection was performed in 2-cell stage embryos in M2 medium using an IMT-2 inverted microscope (Olympus), micromanipulators (Leica Microsystems) and a manual microinjection system. Embryos were co-injected with mRNAs for EsrrbtdTomato $(275$ or $550 \mathrm{ng} / \mu \mathrm{l})$ and $\mathrm{mGFP}(350 \mathrm{ng} / \mu \mathrm{L})$ and cultured in KSOM+AA+ D-Glucose (EmbryoMax, Millipore) for $48 \mathrm{~h}$. No statistical method was used to predetermine sample size; the experiments were not randomized; the investigators were not blinded to allocation during experiments and outcome assessment.

\section{Imaging:}

Culture conditions: Unless otherwise specified, cells were plated on IBIDI hitreat plates coated overnight with Poly-L-ornithine $0.01 \%$ (Sigma, P4957) at $4^{\circ} \mathrm{C}$, washed and coated $2 \mathrm{~h}$ with laminin (Millipore, CC095) $10 \mu \mathrm{g} / \mathrm{ml}$ in PBS. Cells were transferred to red-phenol-free medium before imaging.

Wide-Field Fluorescence and deconvolution: EtdT and Esrrb-GFP cells were incubated with 100nM Hoechst-33342 for $45 \mathrm{~min}$ before imaging (Fig. 1). Alternatively, cells were cultured in $50 \mathrm{ng} / \mathrm{ml}$ 
nocodazole (Sigma, M1404) for 4 hours, with addition of 100nM Hoechst-33342 for 15 min, and mitotic cells collected by shake-off and resuspended in a minimal volume of fresh medium. One drop was deposed on a glass slide and a glass coverslip gently deposed on top. Cells were imaged immediately

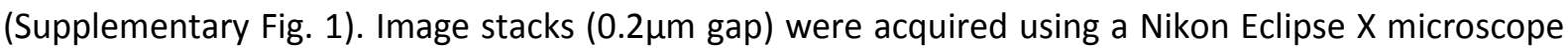
equipped with: $63 x$ oil immersion objective (N.A1.4); LUMENCOR excitation diodes; Hamamatsu ORCAFlash 4.0LT camera; NIS Elements 4.3 software. After photo-bleaching correction (EMBL Plugin CorrectBleach), deconvolution was performed with Volocity 5.4 .0 (99\% confidence and 23max iteration), using calculated PSFs and $0.11 \mu \mathrm{m}$ effective pixel size.

Spinning Disk Fluorescence imaging for FRAP or acquisition of images showing exclusion of Nanog-GFP and Mutant Esrrb-GFP: WT or Mutant Esrrb-GFP EKOiE cells or Nanog-GFP E14Tg2a were kept at $37^{\circ} \mathrm{C}$ in a humidified atmosphere ( $7 \% \mathrm{CO} 2)$ during the entire duration of the experiments. Only for acquisition of images showing exclusion of Nanog-GFP and Mutant Esrrb-GFP, cells were incubated with $100 \mathrm{nM}$ Hoechst-33342 for 15 min before imaging. Images were acquired using a $63 \mathrm{x}$ oil immersion objective on a Zeiss AxioObserver Z1 microscope equipped with Yokagawa CSUX1 spinning-disk confocal setup, a Hamamatsu EMCCD ImageEM X2 camera and Volocity imaging software. For FRAP, 10 frames were acquired before bleaching (20ms pulse using a $488 \mathrm{~nm}$ laser) and then recovery was imaged for $2 \min (1$ image each $110 \mathrm{~ms})$. Fluorescence recovery was analysed in Matlab as described ${ }^{55}$, with one modification: the bleached region was located automatically by subtracting the first prebleach image from the last post-bleach image and calculating the $2 \mathrm{~d}$ convolution (conv2 function) of this image with a circle of the typical size of a bleach spot.

Time-lapse imaging: WT or Mutant Esrrb-GFP EKOiE ES were kept at $37^{\circ} \mathrm{C}$ in a humidified atmosphere containing $7 \%$ CO2 during the entire duration of the movie. Image stacks with a $0.3 \mu \mathrm{m}$ gap were acquired every 3 min using a $63 x$ oil immersion objective (N.A1.4) on a Zeiss Observer Z1 microscope 
(CoolLED excitation system, Photometrics Evolve EMCCD camera, Metamorph software).

Deconvolution was performed as described above with $0.2539 \mu \mathrm{m}$ effective pixel size.

Karyotype staining: $5 \times 10^{6}$ cells were arrested in metaphase with Colcemid (4h; $100 \mathrm{ng} / \mathrm{ml} ; \mathrm{Gibco}$, 15212-012), trypsinised and resuspended in $10 \mathrm{ml}$ of hypotonic solution (NaCitrate $0.017 \mathrm{M}, \mathrm{KCl} 0.03 \mathrm{M}$ ) for $10 \mathrm{mins}$ at RT. The cells were resuspended in $2.5 \mathrm{ml}$ of ice-cold hypotonic solution. $5 \mathrm{ml}$ of cold fixative acetic acid-methanol 1:3 solution were added dropwise at $4^{\circ} \mathrm{C}$. Cells were collected and resuspended in $2.5 \mathrm{ml}$ of the supernatant and the above procedure was repeated 3 times. Fixed cells were spread by dropping on pre-heated glass slides. Slides were incubated in PBST (PBS 0.1\% Triton X100; 10 mins), blocked in PBST/5\% donkey serum (30 mins) and incubated (2h, RT) with primary antibodies (1:500 Esrrb from Perseus Proteomics H6-705-00 and/or 1:200 Nanog from Cosmobio RCAB0001P). After 3 washes with PBST, samples were incubated with goat anti-mouse or anti-rabbit secondary antibody (1:500; Alexa 488 or 594; Jackson ImmunoResearch, 715-545-151; 715-585-150; 711-545-152) over-night at 4으, washed, mounted (Vectashield; VectorLab, H1200) and imaged as for wide-field fluorescence.

EKOiE cells ectopically expressing a SNAP-Esrrb fusion protein were cultured in the presence of 150nM SNAP-Cell TMR-Star (NEB, S9105S) over-night, washed and cultured for $2 \mathrm{~h}$ in medium without TMRStar before karyotype staining.

Imaging of embryos: To prevent blastomere mitotic exit, microinjected embryos were incubated with $1 \mu \mathrm{M}$ MG132 6 hours prior to imaging. After 30min in $1 \mu \mathrm{g} / \mathrm{ml}$ Hoechst-33342, live imaging was performed in microdrops of KSOM with 10mM HEPES and $1 \mu \mathrm{M}$ MG132 on glass-bottomed microwell dishes (MatTek) using a Leica TCS SP5 confocal microscope (700Hz, HC Plan Apochromat 20x/0.70NA immersion objective, 25\% laser intensity, voxel size of $75 \mathrm{~nm}$, pinhole of 1 airy unit). 


\section{Preparation of mitotic populations:}

E14tg2a or EKOiE cells were grown to $70-80 \%$ confluency in 4 to 6 T150 flasks per preparation. Vigorous shake off was performed to detach debris, dying cells or poorly attached cells colonies, medium discarded and flasks washed twice in PBS. After 4 hours in medium containing $50 \mathrm{ng} / \mathrm{ml}$ nocodazole (Sigma, M1404), flasks were gently washed with PBS. Gentle shake-off was performed in $10 \mathrm{ml}$ of medium, monitoring the process under a microscope to avoid detaching clumps of cells in interphase. The cell suspension was filtered through a double layer of $20 \mu \mathrm{m}$ filters (Millipore, NY2004700) and spun down. Typically, $10^{7}$ cells were obtained from each preparation. Purity of mitotic preparations were systematically checked under a fluorescence microscope after fixation, cytospinning and DAPI staining. Preparations with more than 5\% contamination from interphase were discarded.

\section{Chromatin preparation for Esrrb binding analysis:}

In previous reports ${ }^{56}$ describing Esrrb ChIP analysis a dual crosslinking strategy with DSG and FA was used. Therefore, we initially applied similar conditions. However, in order to prove that Esrrb binding is not necessarily indirect, as it may be the case when DSG is required, we established an alternative protocol using FA only and digested chromatin with MNase rather than by sonication. Similar ChIP-Seq profiles were observed (Bioinformatic analyses section).

Chromatin preparation (DSG-FA-Sonication): $10^{7}$ ES cells were cross-linked in $2 \mathrm{ml}$ of freshly prepared PBS-DSG $2 \mathrm{mM}$ at pH7.0 (Sigma, 80424-5mg) for $50 \mathrm{~min}$ at RT with occasional shaking. After pelleting and washing in PBS, cells were incubated $10 \mathrm{~min}$ in $2 \mathrm{ml} \mathrm{PBS} \mathrm{1 \%} \mathrm{formaldehyde} \mathrm{(Thermo,} \mathrm{28908).} \mathrm{Cross-}$ linking was stopped with $0.125 \mathrm{mM}$ glycine for $5 \mathrm{~min}$ at RT. Cells were pelleted, washed with ice-cold PBS and resuspended in $2 \mathrm{ml}$ of swelling buffer ( $25 \mathrm{mM}$ Hepes pH7.95, 10mM KCl, 10mM EDTA) freshly 
supplemented with 1X protease inhibitor cocktail (PIC-Roche, 04693116 001) and 0.5\% NP-40. After 30 min on ice, suspension was passed 50 times in a dounce (only for asynchronous populations), centrifuged and resuspended in $300 \mu$ of TSE150 (0.1\% SDS, 1\% Triton, 2mM EDTA, 20mM Tris-HCl pH8, $150 \mathrm{mM} \mathrm{NaCl}$ ) buffer, freshly supplemented with $1 \mathrm{X}$ PIC. Samples were sonicated in $1.5 \mathrm{ml}$ tubes (Diagenode) using a Bioruptor Pico (Diagenode) for 7-9 cycles divided into $30 \mathrm{sec}$ ON-30 sec OFF subcycles at maximum power, in circulating ice-cold water. After centrifugation ( $30 \mathrm{~min}$, full speed, $4^{\circ} \mathrm{C}$ ), the supernatant was stored at $-80^{\circ} \mathrm{C} .5 \mu \mathrm{l}$ were used to quantify the chromatin concentration and check DNA size (typically 200-500bp).

Chromatin Preparation (FA-Mnase): $10^{7}$ ES cells were resuspended in $3 \mathrm{ml}$ of pre-warmed DMEM/FCS/LIF and cross-linked for 10min at RT adding $1 \%$ formaldehyde (Thermo, 28908). Cells were processed as above until nuclei were centrifuged, resuspended in $500 \mu \mathrm{l}$ of $\mathrm{KN}$ buffer $(20 \mathrm{mM}$ Tris- $\mathrm{HCl}$ PH7.5, $15 \mathrm{mM} \mathrm{KCl}, 60 \mathrm{mM} \mathrm{NaCl}, 0.34 \mathrm{M}$ Sucrose, $1 \mathrm{mM} \mathrm{CaCl}$ ) and $60 \mathrm{U}$ MNase (Thermo, EN0181). After $7 \mathrm{~min}$ at $37^{\circ} \mathrm{C}$, the reaction was stopped on ice with $4 \mu \mathrm{l}$ of $500 \mathrm{mM}$ EDTA. Samples were sonicated in ice-cold water (Bioruptor, Diagenode; 4 cycles of $20 \mathrm{sec}$ ON-40 sec OFF, maximum power). The chromatin was centrifuged $\left(30 \mathrm{~min}\right.$, full speed, $4^{\circ} \mathrm{C}$ ) and stored at $-80^{\circ} \mathrm{C}$ until use. $5 \mu \mathrm{l}$ were used to quantify the chromatin and check DNA size (typically a highly prevalent mono-nucleosome band).

\section{Chromatin Immuno-Precipitation (ChIP):}

For ChIP-qPCR and ChIP-Seq experiments chromatin from $2.10^{6}$ and $10^{7}$ cells was used respectively. Chromatin was pre-cleared for $90 \mathrm{~min}$ rotating on-wheel at $4^{\circ} \mathrm{C}$ in $300 \mu \mathrm{l}$ of TSE150 containing $50 \mu \mathrm{l}$ of pG sepharose beads (Sigma, P3296-5ML) 50\% slurry, previously blocked with BSA (500 $\mu \mathrm{g} / \mathrm{ml}$; Roche, 5931665103) and yeast tRNA (1 $\mu \mathrm{g} / \mathrm{ml}$; Invitrogen, AM7119). Immunoprecipitations with anti-Esrrb 
mouse monoclonal (Perseus Proteomics, H6-705-00) $\left(1 \mu \mathrm{g} / 2.10^{6}\right.$ cells) were performed overnight rotating on-wheel at $4^{\circ} \mathrm{C}$ in $500 \mu \mathrm{l}$ of TSE150. $20 \mu \mathrm{l}$ were set apart for input DNA extraction and precipitation. $25 \mu \mathrm{l}$ of blocked pG beads $50 \%$ slurry were added for $4 \mathrm{~h}$ rotating on wheel at $4^{\circ} \mathrm{C}$. Beads were pelleted and washed for $5 \mathrm{~min}$ rotating on-wheel at RT with $1 \mathrm{ml}$ of buffer in the following order: 3xTSE150, 1xTSE500 (as TSE150 but 500mM NaCl), 1x washing buffer (10mM Tris-HCl pH8, 0.25M LiCl, 0.5\% NP40, 0.5\% Na-Deoxycholate, 1mM EDTA), and 2xTE (10mM Tris-HCl pH8, 1mM EDTA). Elution was performed in $100 \mu \mathrm{l}$ of elution buffer ( $1 \%$ SDS, $10 \mathrm{mM}$ EDTA, $50 \mathrm{mM}$ Tris- $\mathrm{HCl} \mathrm{pH} 8$ ) for $15 \mathrm{~min}$ at $65^{\circ} \mathrm{C}$ after vigorous vortexing. Eluates were collected after centrifugation and beads rinsed in $150 \mu$ l of TESDS1\%. After centrifugation, the supernatant was pooled with the corresponding first eluate. For both immunoprecipitated and input chromatin, the crosslinking was reversed overnight at $65^{\circ} \mathrm{C}$, followed by proteinase $\mathrm{K}$ treatment, phenol/chlorophorm extraction and ethanol precipitation.

RNA isolation and Reverse Transcription:

Total RNA was isolated in Trizol (Invitrogen, 15596026) and digested with DNase I (Qiagen, 79254). Reverse transcription reactions were performed with random hexamers on $1 \mu \mathrm{g}$ of total RNA (First Strand cDNA kit, Roche, 04379012001).

\section{Quantitative real-time PCR:}

Real-time PCR reactions were performed in quadruplicate for pre-mRNA expression analysis and duplicate for ChIP analysis in 384-wells plates with a 480 LightCycler (Roche) using LightCycler 480 SYBR Green I Master (Roche, 04707516001). Standard and melting curves were generated to verify the amplification efficiency (>85\%) and the production of single DNA species. PCR primer sequences are 
listed in Supplementary Information Table 4. Values for gene expression were normalised to the levels of Tbp mRNA.

FACS:

Ccna-GFP EKOiE ES cells were incubated with $20 \mu \mathrm{M}$ Hoechst 33342 for $45 \mathrm{~min}$, filtered through a $40 \mu \mathrm{m}$ cell strainer and kept on ice. ES cells were sorted based on Hoechst and GFP levels in 3 populations (EarlyG1, LateG1 and G2) using a FacsARIA III (Becton-Dickinson), keeping samples and collection tubes at $4^{\circ} \mathrm{C}$. Data was analysed using the FlowJo software suite (Tree Star). Around $10^{6}$ cells were sorted per population before RNA extraction. For replating experiments, sorted cells were cultured for 7 hours, incubated 45 minutes with Hoechst, trypsinised and analysis with a LSR II Fortessa (Becton-Dickinson).

\section{Bioinformatic analyses:}

ChIP-Seq sequencing and alignment: ChIP-Seq libraries (Supplementary Information Table 5) were generated using a MicroPlex kit (Diagenode) and sequenced (50bp; HiSeq 2500 instrument, Illumina). Reads were mapped to the $\mathrm{mm} 9$ genome assembly using Bowtie and allowing 3 mismatches using GeneProf ${ }^{57}$. Aligned reads corresponding to Interphase, Mitosis and respective controls were separately pooled. Unless otherwise specified all analyses were performed with SeqMonk (http://www.bioinformatics.babraham.ac.uk/projects/seqmonk/). We generated 500bp-long windows sliding every $250 \mathrm{bp}$ and quantitated read counts per kb per million (RPKM) across the genome after excluding the $\mathrm{Y}$ chromosome (lost in EKOiE). Interphase_rep4 was a clear outlier in terms of fold-coverage and global correlation to other interphase replicates (Supplementary Information Table 5). 
Identification of candidate peaks and statistical analysis: Peaks were called with MACS as implemented in SeqMonk using EKOiE -dox controls as background, a $10^{-5} \mathrm{p}$-value threshold, and fragment sizes of 200, 350 and 300 for MNase, sonicated and pooled datasets respectively. Candidate peaks from pooled datasets (excluding Y chromosome) were combined and subject to a DESEQ2 statistical filter using raw read counts of each individual replicate (excluding Interphase_rep4). Multiple testing correction and independent filtering were enabled leading to 20.151 peaks (FDR $<0.2$ in Interphase or Mitosis versus the -dox controls). Peaks were simplified such that no two peaks overlap by more than $1 \%$, giving 14.559 binding regions.

Categorisation of DESEQ2 filtered peaks as Bookmarked or Lost regions: 14.559 regions passing DESEQ2 filter were divided in 5 classes (Fig. S3) according to statistical significance of their enrichment levels (versus -Dox) and the reproducibility of their identification in individual replicates:

Class 1 (450 peaks): Passed DESEQ only in Mitosis. However, most (437) were identified as peaks in at least two Interphase replicates and all but one were identified in at least one of our Interphase replicates or in an independent study ${ }^{3}$. Therefore, these peaks cannot be rigorously qualified as mitotic-specific. ChIP-qPCR on 3 Class_1 peaks, including the single peak that was not detected in any Interphase replicate (Fig. S4), confirmed this interpretation.

Class 2 (1216): Passed DESEQ filter both in Mitosis and in Interphase. They represent regions bound by Esrrb in Interphase and in Mitosis.

Class 3 (314): Passed DESEQ only in Interphase but were identified as peaks in all 4 Mitosis replicates. These regions are characterised by robust Esrrb binding in Interphase and low but reproducible binding in Mitosis. 
Class 4 (6621): Passed DESEQ only in Interphase and were identified as peaks in the pooled Mitosis dataset but not in all Mitosis replicates.

Class 5 (5958): Passed DESEQ only in Interphase and were never detected in any of the Mitosis replicates. They represent clear Interphase-specific Esrrb binding regions.

Although Class_4 regions may represent weak binding in Mitosis, we followed a conservative approach and combined them with Class_5 regions into the list of Lost regions. In contrast, Classes_1,2,3 represent regions that bind Esrrb in Mitosis and Interphase (although at different degrees, see Figs. S3 and S4); they were combined into the list of Bookmarked regions. Coordinates and Class of each individual peak, together with the quantifications in all datasets are available in Table S1.

ChIP-Seq binding profiles: The ChIP-Seq profiles shown in Fig.2 and Fig. S4 were obtained in SeqMonk designing $100 \mathrm{bp}$ windows every $10 \mathrm{bp}$ and quantitating the base coverage normalised to the total number of aligned reads of the pooled datasets. A smoothing window of 25 was then implemented. The profiles shown in Supplementary Fig. 5 were obtained similarly but using 100bp windows sliding every 50bp.

Average profiles: All metaplots were calculated in SeqMonk using base coverage per million reads every $100 \mathrm{bp}$. All Esrrb peaks overlapping with a +/-500bp region flanking mRNA 5'-ends were considered promoters, leaving the rest as enhancers.

Hierarchical clustering and heatmaps: The heatmaps shown in Fig. 3 and Fig. S3 were obtained in SeqMonk (RPKM quantification) using either $5 \mathrm{~kb}$-long regions centred on the middle of each peak or the exact peak coordinates, respectively. To generate B1, B2, L1 and L2 groups a euclidean clustering based on the smallest absolute difference between region quantification values was used. 
Expression of genes associated to Esrrb binding sites: All 4 clusters of binding regions identified by hierarchical clustering (Fig. 3) were used as inputs for GREAT ${ }^{58}$ using standard 'basal plus extension' parameters. The first 5 tissues showing expression of the selected genes according to the Mouse Gene Informatics database are shown in Fig. 3c. In Fig. 3d all genes located within 25kb of each Esrrb-binding region were selected. If a gene was near two or more peaks belonging to distinct clusters, it was associated to one only in a prioritized manner (B1>B2>L1>L2). Next, RPKM values were calculated in several datasets corresponding to nuclear RNA-Seq, Ser2-phosphorylated RNAPII and H3K36me3.

De-novo motif discovery: The RSAT peak-motif tool ${ }^{59}$ was used on a virtual machine (rsat-vm-2015-10) to identify motifs enriched at Bookmarked or Lost regions using 6, 7 or 8bp-long seeds on both strands. The significance of the motifs was established using a background model of Markov order adapted to the length of the sequences. The corresponding TFs were identified on cisBP and JASPAR databases as implemented in RSAT. To search for putative Esrrb binding sites in all the regions a background model was used with a Markov order of 1.

RNA-seq sequencing and alignments: Libraries for RNA-Seq (Supplementary Information Table 5) were prepared using the TruSeq Stranded mRNA Sample preparation kit (Illumina) with rRNA-depleted samples (Ribo-Zero Gold kit - Illumina). Strand-specific sequencing (50bp) was performed on a HiSeq 2500 instrument (Illumina). Reads were aligned to the $\mathrm{mm} 9$ genome using Bowtie (allowing 50 hits per read; Supplementary Information Table 5).

RNA-Seq quantification and identification of differentially expressed genes: Read counts were quantified as RPKM in SeqMonk. High Pearson correlations were observed between all datasets 
(Supplementary Information Table 5). BAM/SAM files were generated for each aligned library and used as inputs for the iRNASeq pipeline ${ }^{60}$. Raw read counts of exons (mRNA) and introns (pre-mRNA) were normalised and compared using edgeR as implemented in iRNASeq. Fold changes and associated FDR derived from multiple testing were generated for each annotated mRNA and pre-mRNA. We considered genes as upregulated in a given cell-cycle phase if displaying an exonic FDR $<0.1$, an exonic mRNA FC $>0.5$ and an intronic pre-mRNA FC $>0.3$; for downregulated genes exonic $\mathrm{FC}<-0.5$, intronic $F C<-0.3$ and exonic FDR $<0.1$. From genes up/down-regulated in $\mathrm{G} 2$ we subselected those displaying a $-0.6<F C<0.6$ and FDR $>0.1$ in both early and late G1 to identify genes responding to Esrrb levels in G2 only.

Correlation with Esrrb-binding regions: A TAD (http://chromosome.sdsc.edu/mouse/hic/download.html) was considered bookmarked when it included at least one Bookmarked peak. The fraction of Esrrb-responsive genes at each cell-cycle phase located in a bookmarked TADs or located within $25 \mathrm{~kb}$ of a bookmarked peak was scored and compared to randomly selected genes ( 20 lists of 200 genes).

\section{Statistics and Reproducibility:}

The retention of Esrrb on mitotic chromosomes has been systematically observed by independent researchers (N.F, E.G.T, A.D and A.M). Statistical analyses of genome-wide studies were conducted with DESeq, EdgeR and Prism 5.00. Normality was assessed with D'agostino and Pearson test followed by one-way ANOVA using Kruskal-Wallis and Dunn's Multiple Comparison tests or Student t-tests. Contingency tables were analysed with a Fisher's exact test. 


\section{Data availability:}

Primary NGS datasets have been deposited at Geneprof (gpXP_003043) and at the Gene Expression Omnibus (GEO) under accession number GSE75066. Previously published datasets are available under accession numbers GSE11431, GSE29218, GSE34518, GSE23943 and GSE57092. ChIP-Seq and RNA-Seq results as well as all primer sequences used in this study have been provided in Supplementary Tables 1-5. All other data supporting our conclusions as well as the reagents that were generated are available upon reasonable request to P.N. 


\section{References}

54. Gearhart, M.D., Holmbeck, S.M., Evans, R.M., Dyson, H.J. \& Wright, P.E. Monomeric complex of human orphan estrogen related receptor-2 with DNA: a pseudo-dimer interface mediates extended half-site recognition. J Mol Biol 327, 819-832 (2003).

55. Mueller, F., Karpova, T.S., Mazza, D. \& McNally, J.G. Monitoring dynamic binding of chromatin proteins in vivo by fluorescence recovery after photobleaching. Methods Mol Biol 833, 153-176 (2012).

56. van den Berg, D.L. et al. Estrogen-related receptor beta interacts with Oct4 to positively regulate Nanog gene expression. Mol Cell Biol 28, 5986-5995 (2008).

57. Halbritter, F., Vaidya, H.J. \& Tomlinson, S.R. GeneProf: analysis of high-throughput sequencing experiments. Nat Methods 9, 7-8 (2012).

58. McLean, C.Y. et al. GREAT improves functional interpretation of cis-regulatory regions. Nat Biotechnol 28, 495-501 (2010).

59. Medina-Rivera, A. et al. RSAT 2015: Regulatory Sequence Analysis Tools. Nucleic Acids Res 43, W50-56 (2015).

60. Madsen, J.G. et al. iRNA-seq: computational method for genome-wide assessment of acute transcriptional regulation from total RNA-seq data. Nucleic Acids Res 43, e40 (2015). 


\section{Figure 1}

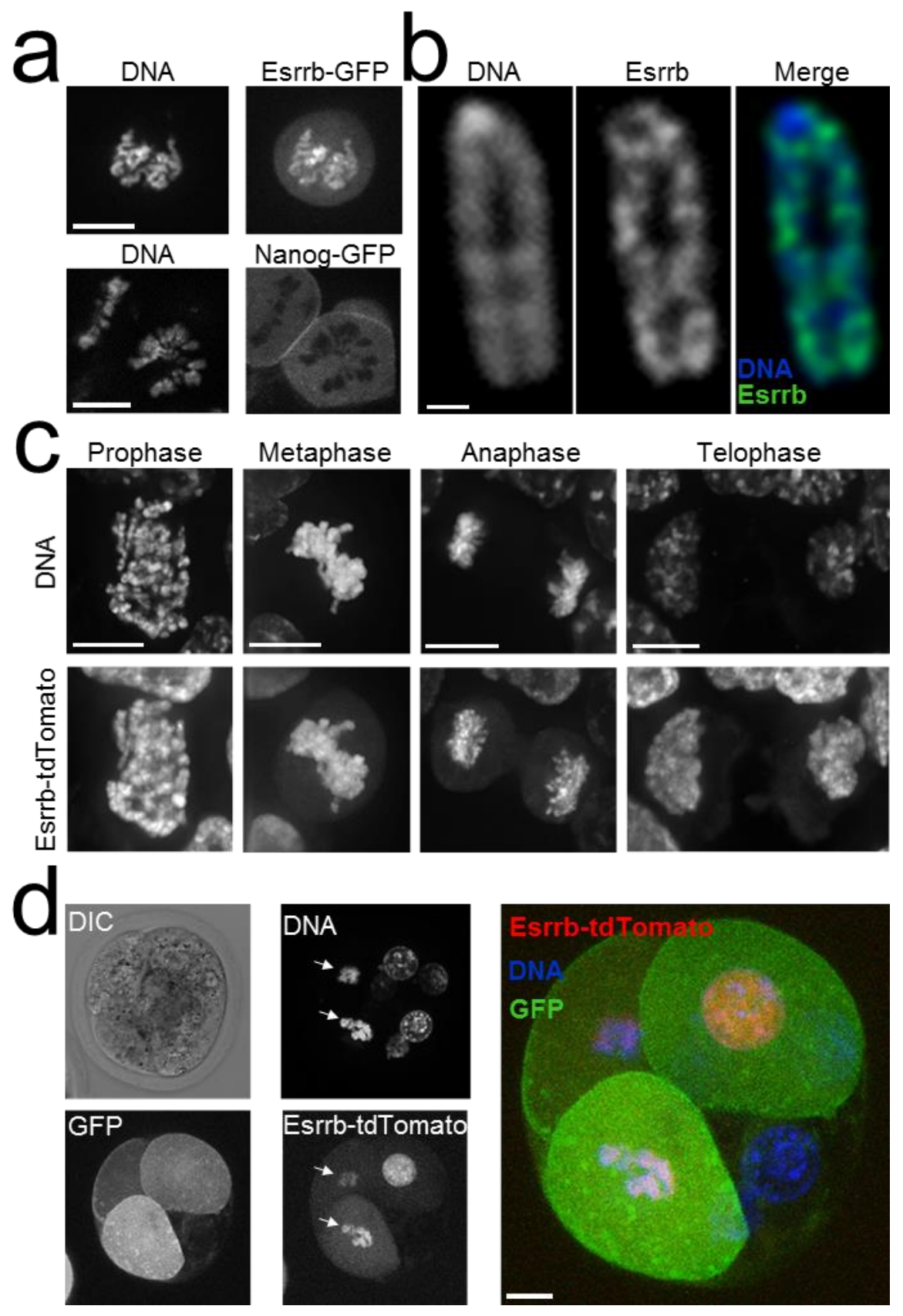


Figure 2

a

$150 \mathrm{~kb}$

Super Enhancer
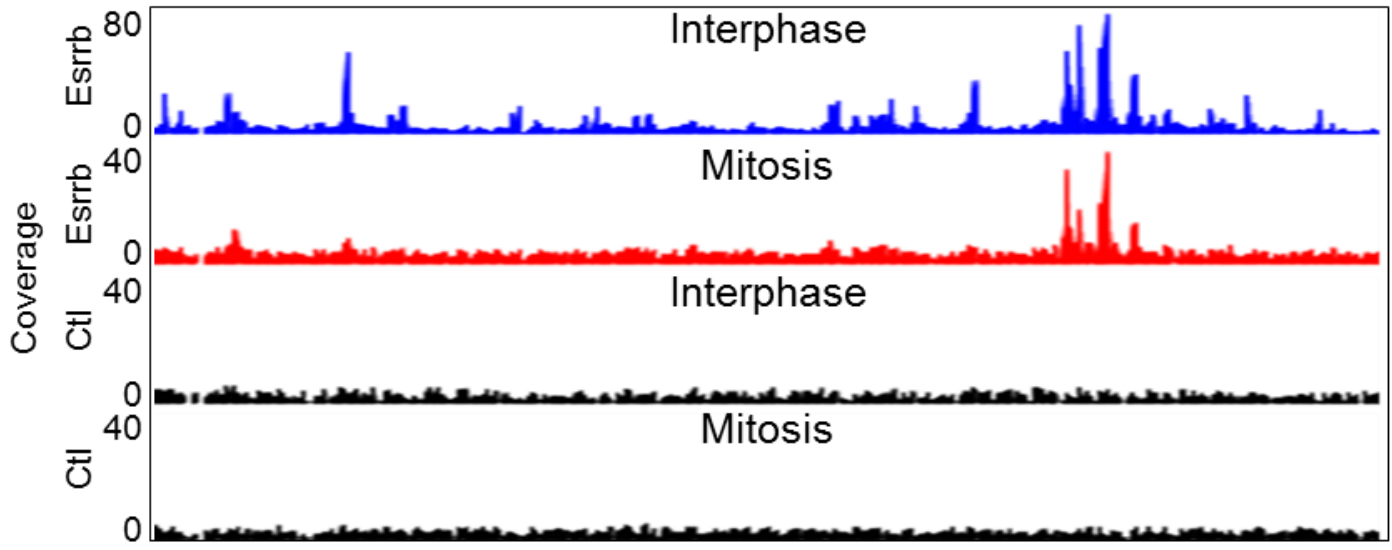

b

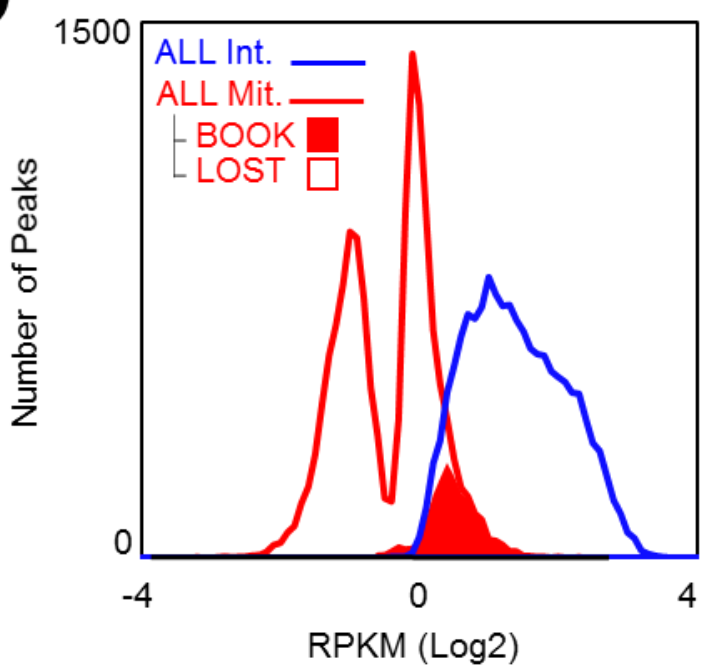

C

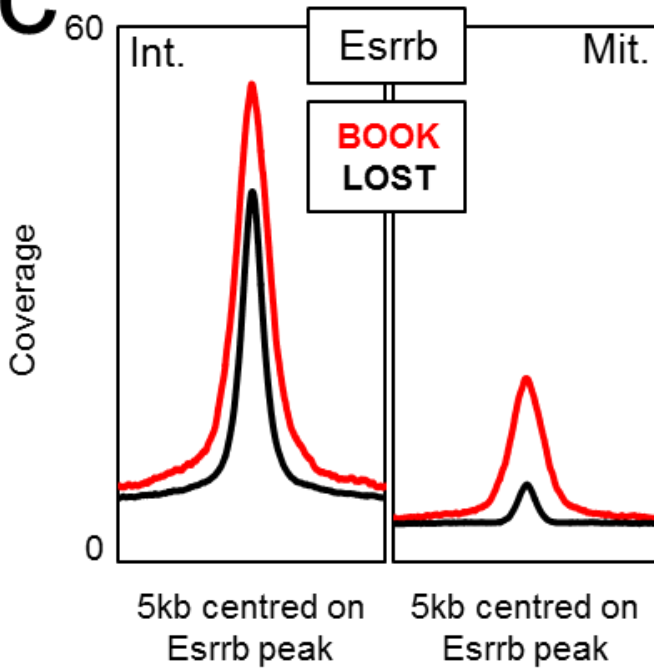

d
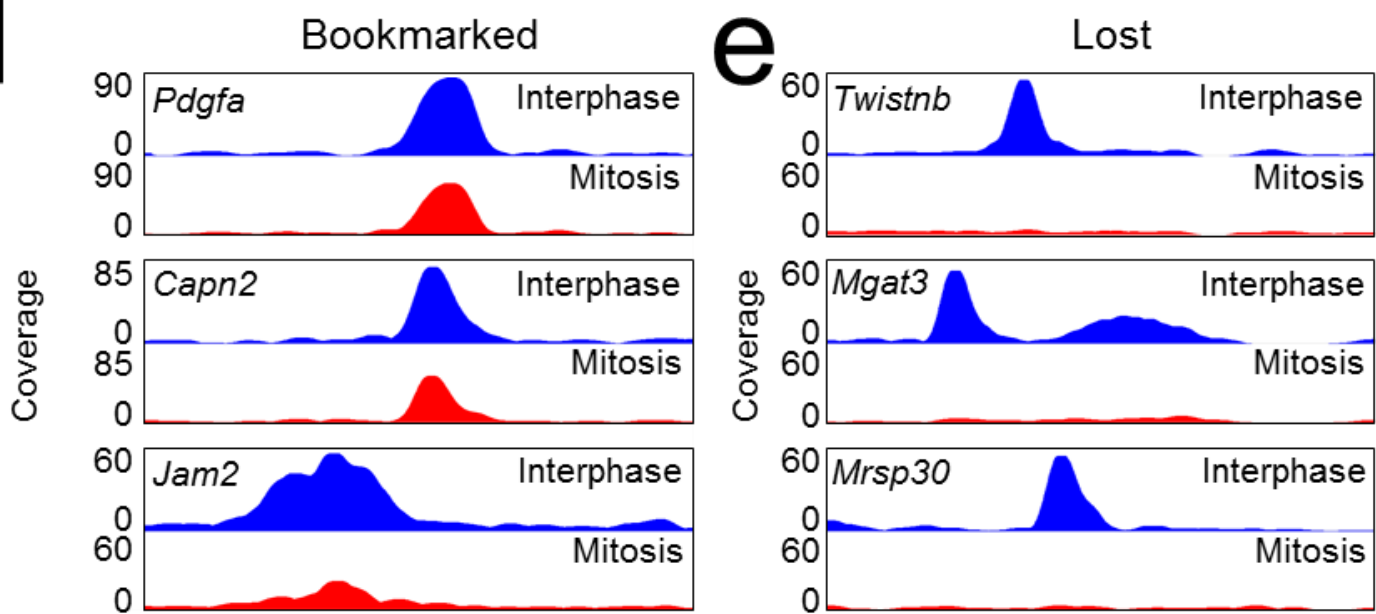


\section{Figure 3}
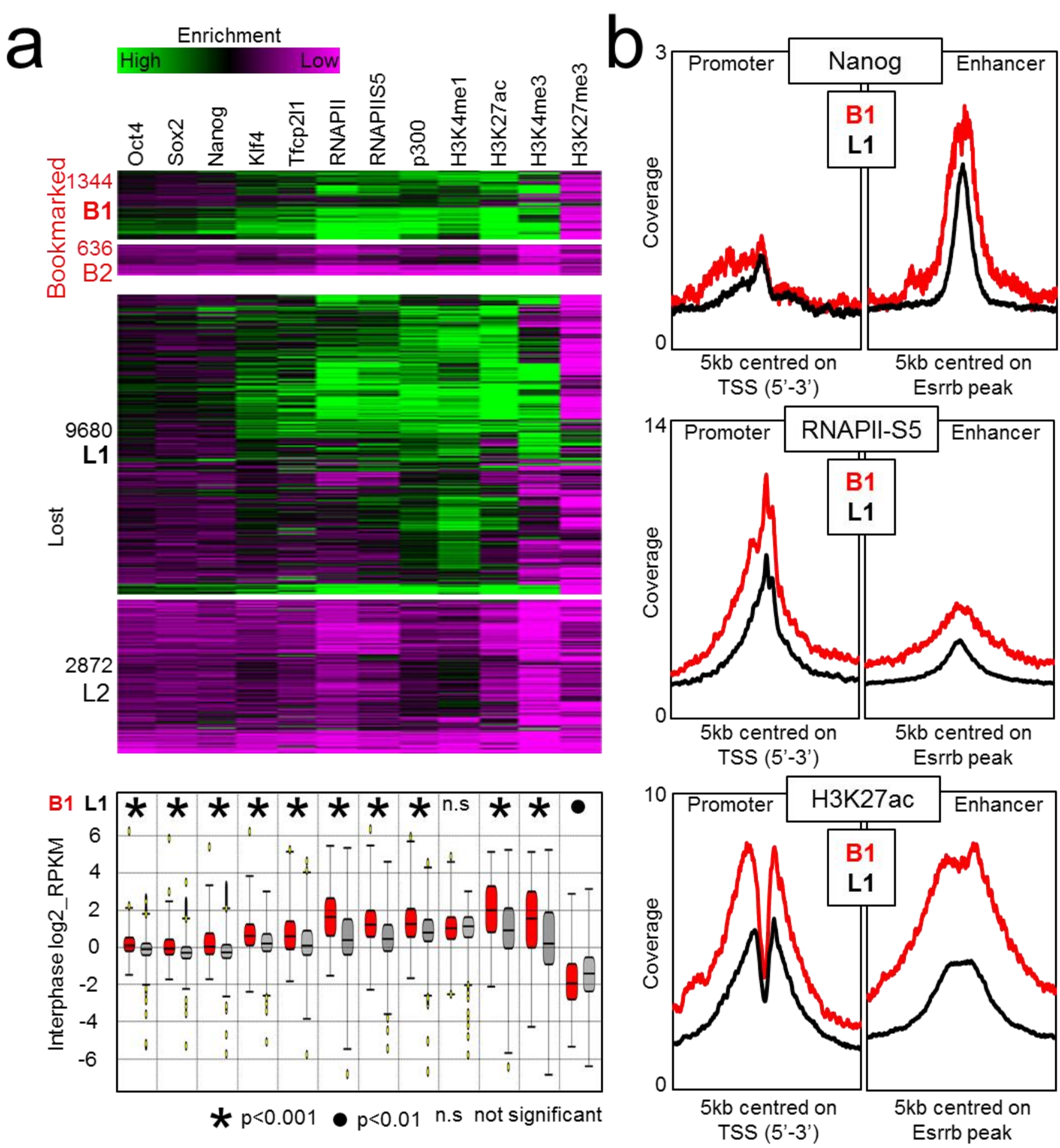


\section{Figure 4}

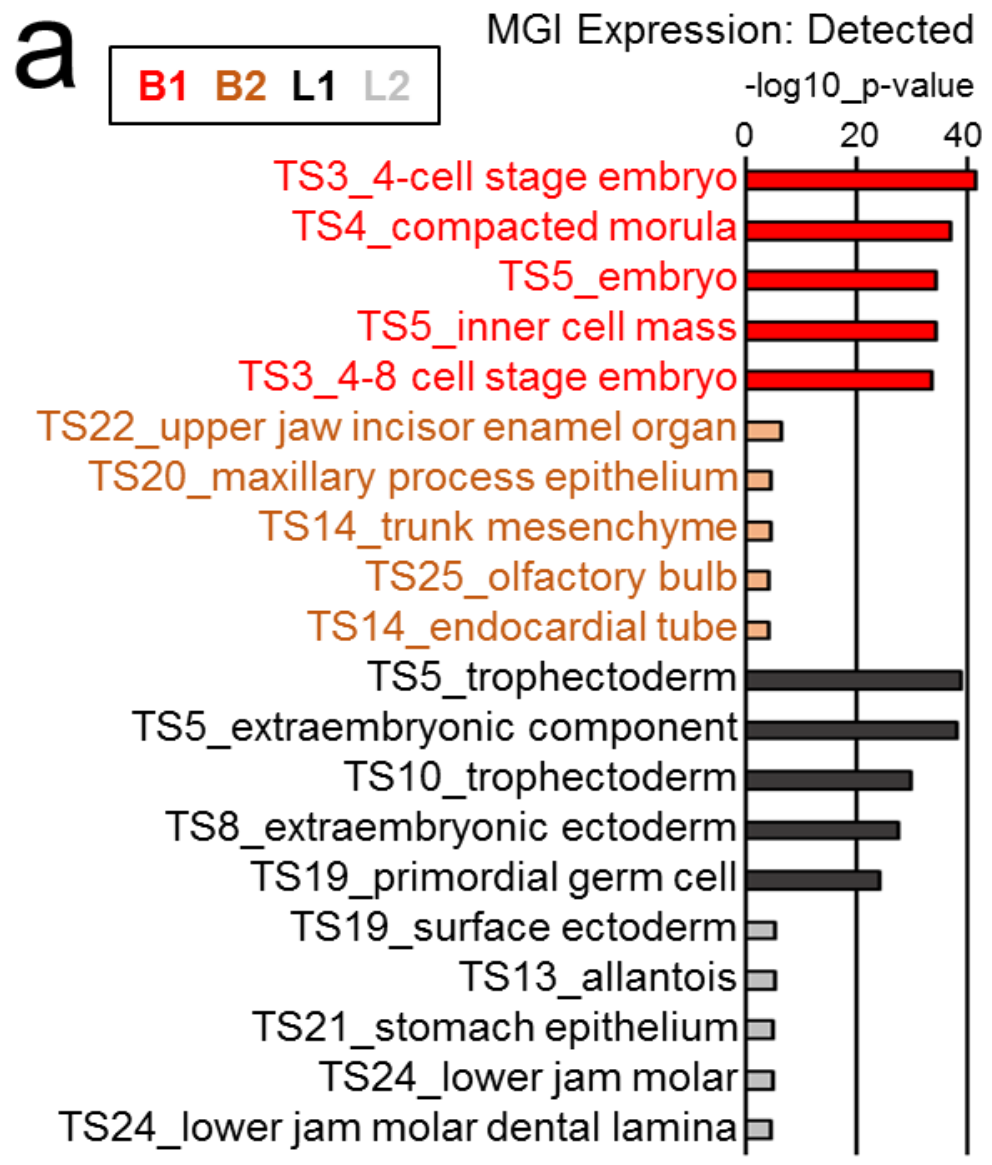

b

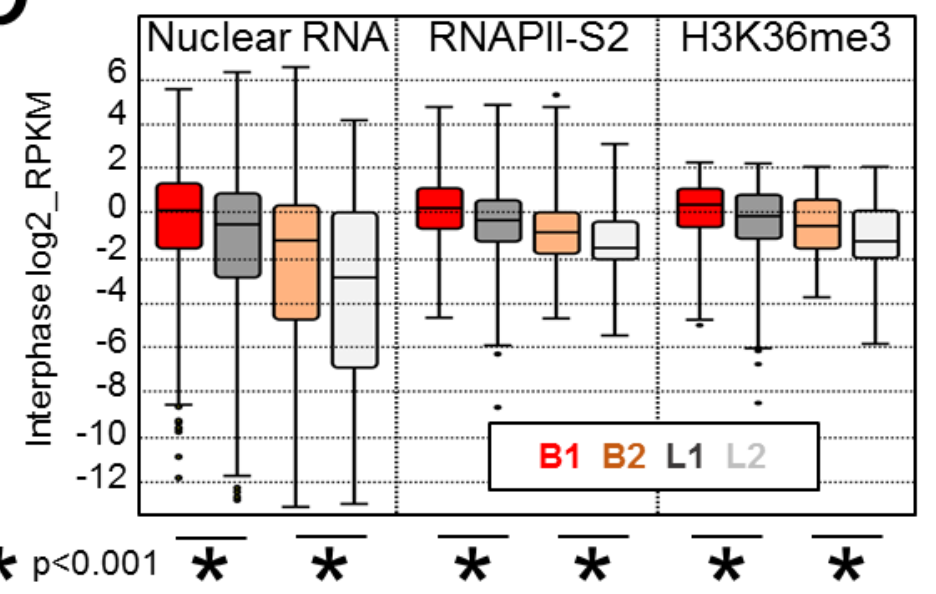




\section{Figure 5}

a

Known Esrrb consensus

Short Motif discoverd in Lost regions

Long Motif discoverd in $\frac{\mathscr{g}}{\underline{0}}$ Bookmarked regions
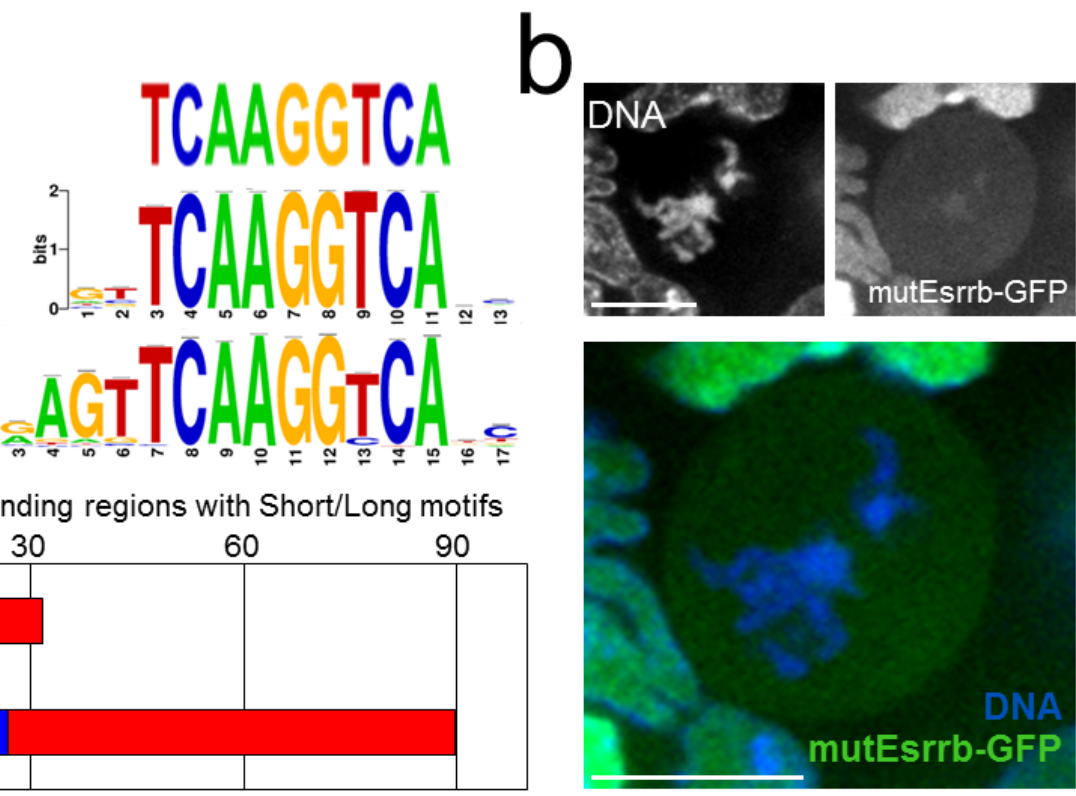

Long Motif $\square \quad \%$ of Esrrb binding regions with Short/Long motifs

$$
\begin{aligned}
& \text { Short } \\
& \text { Bookm }
\end{aligned}
$$

Lost
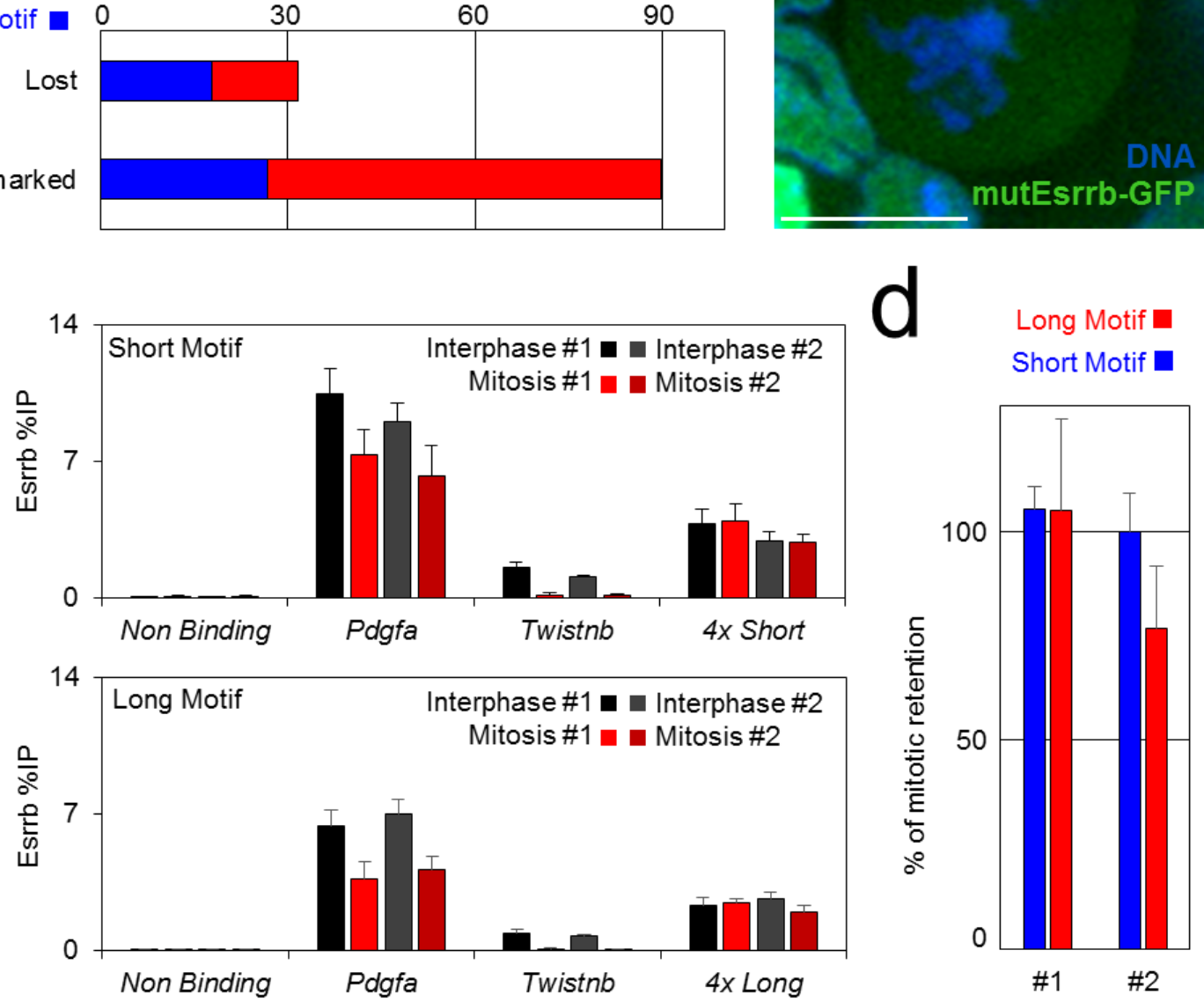


\section{Figure 6}
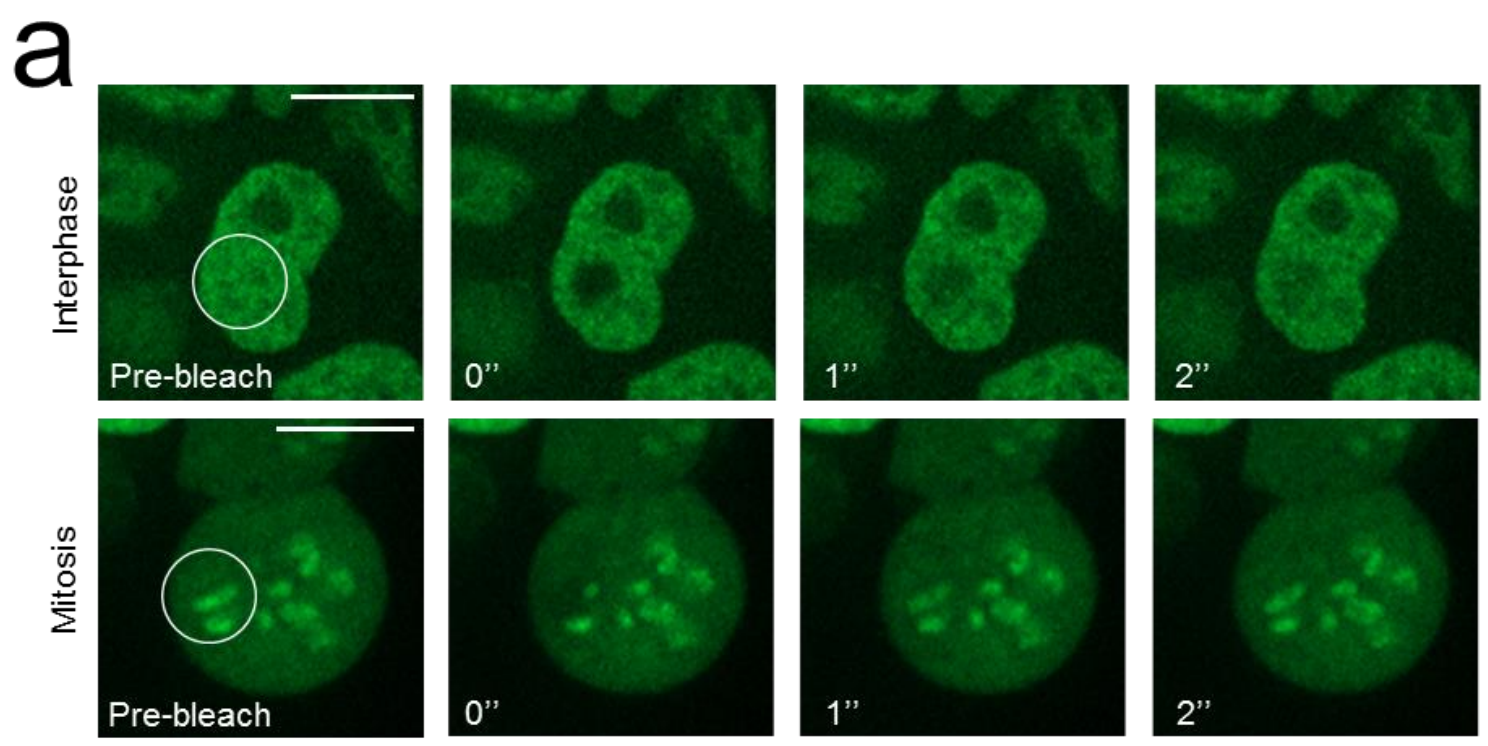

b
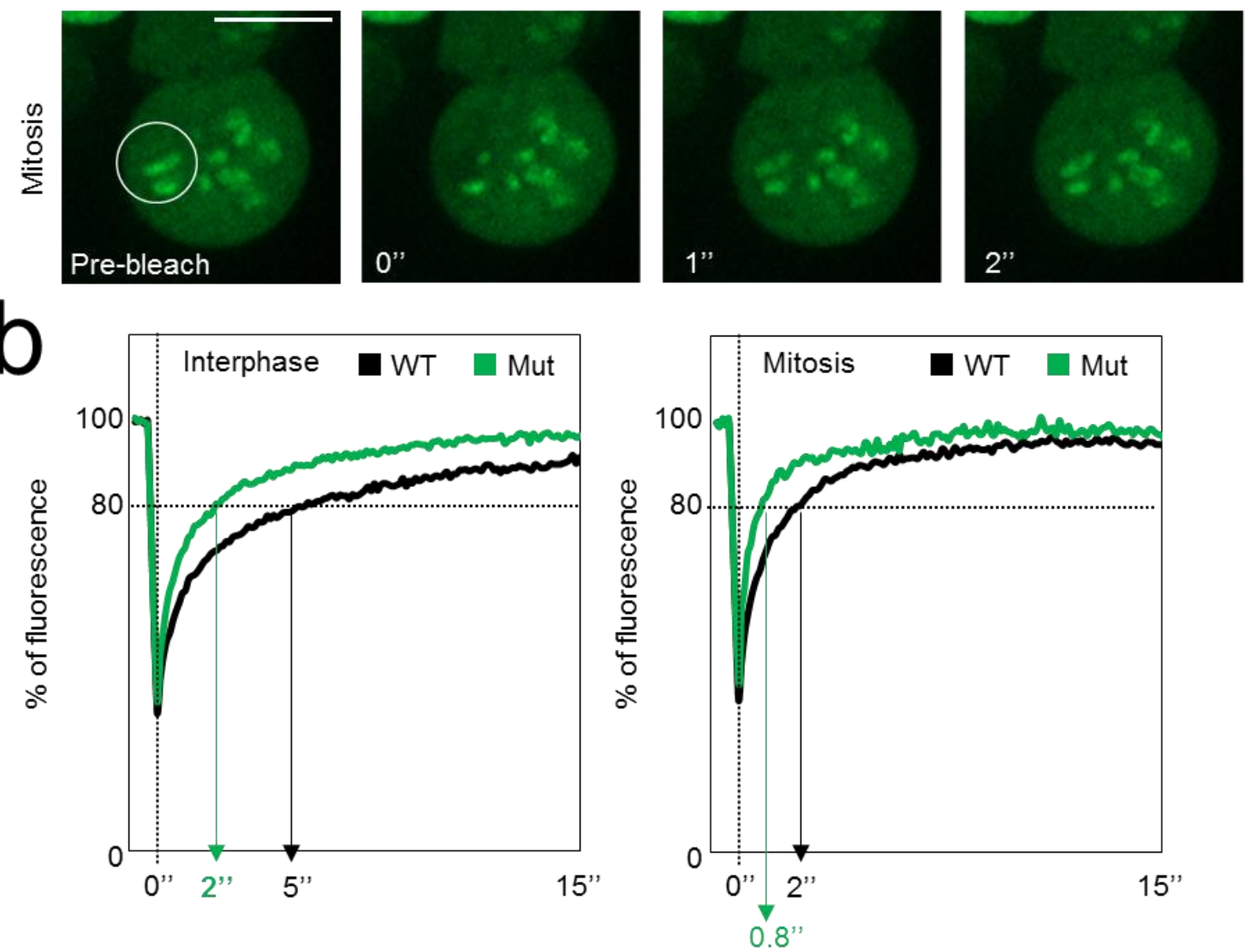
Figure 7

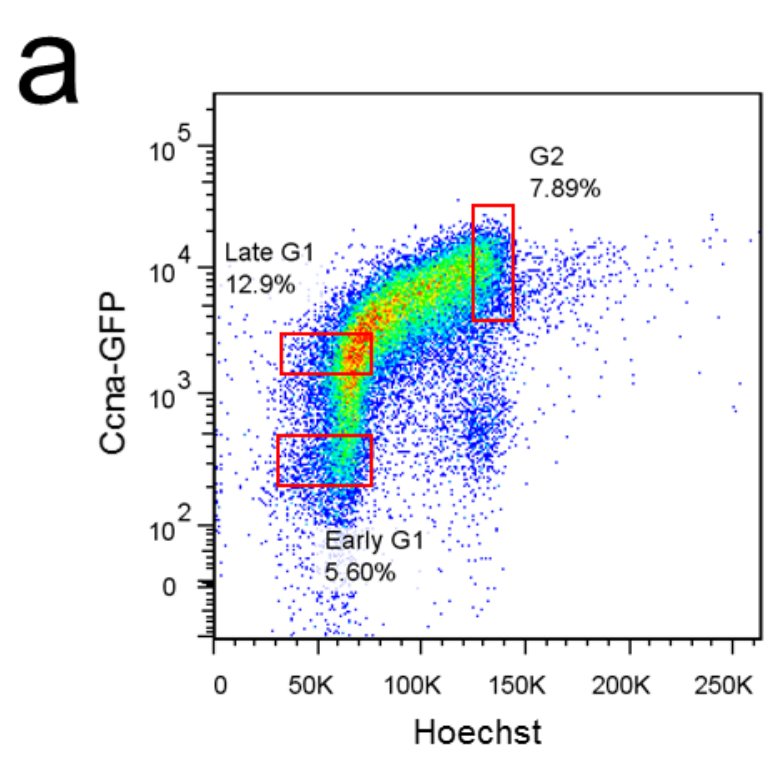

b

Up-regulated genes in:

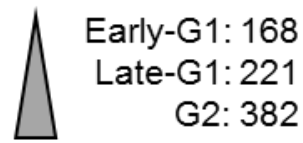

Down-regulated genes in:

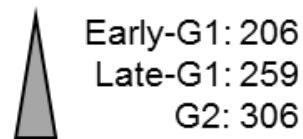

C

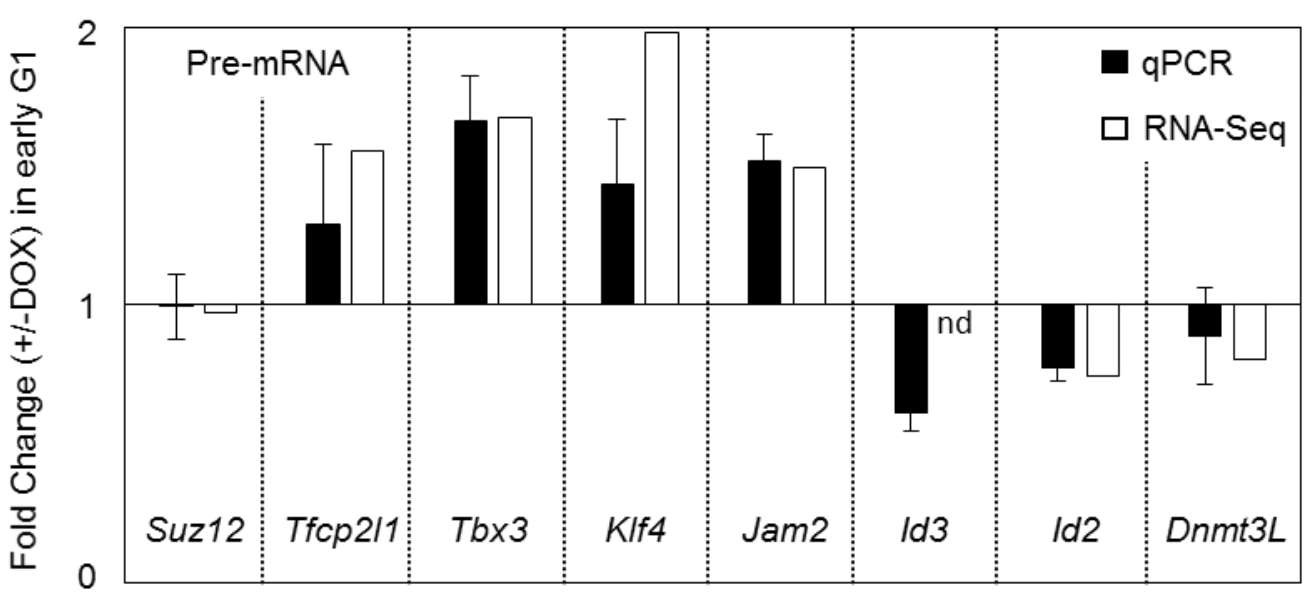

d

100

Bookmarked TADs

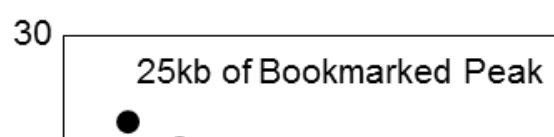

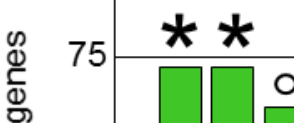

*

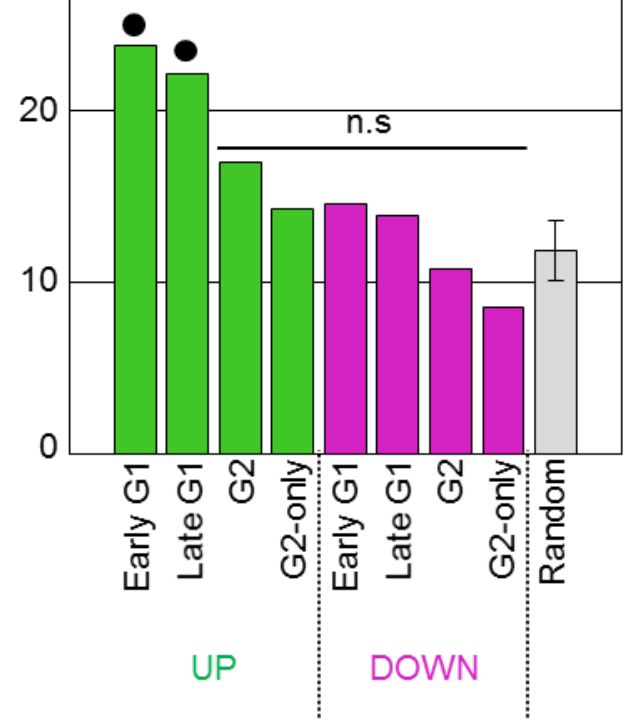

$\star p<0.001$

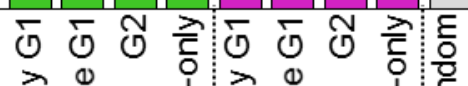

$\mathrm{p}<0.01$

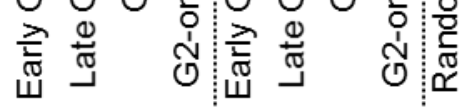

$O p<0.05$

UP

DOWN 
Figure 8
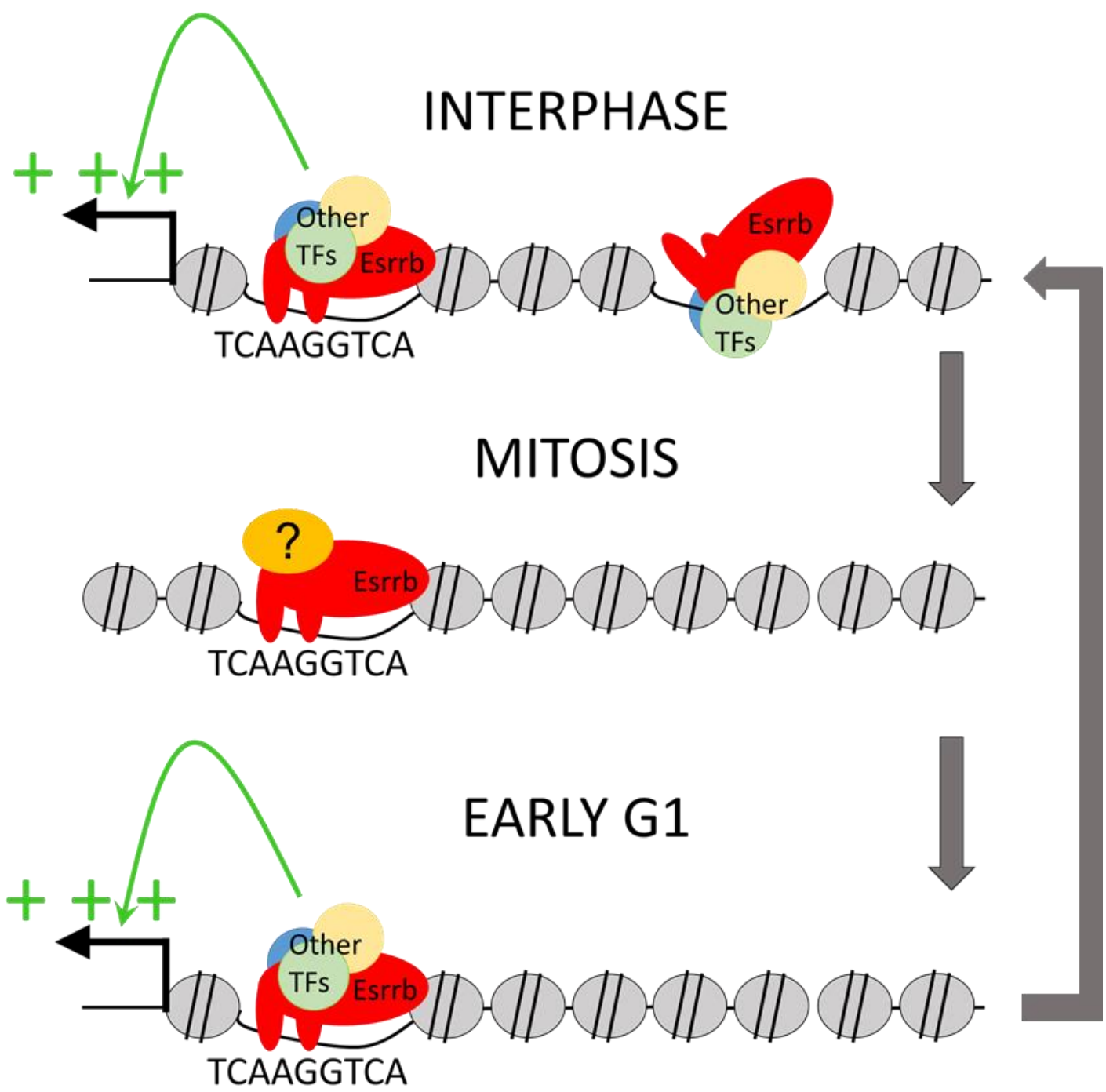


\section{Supplementary Figure 1}
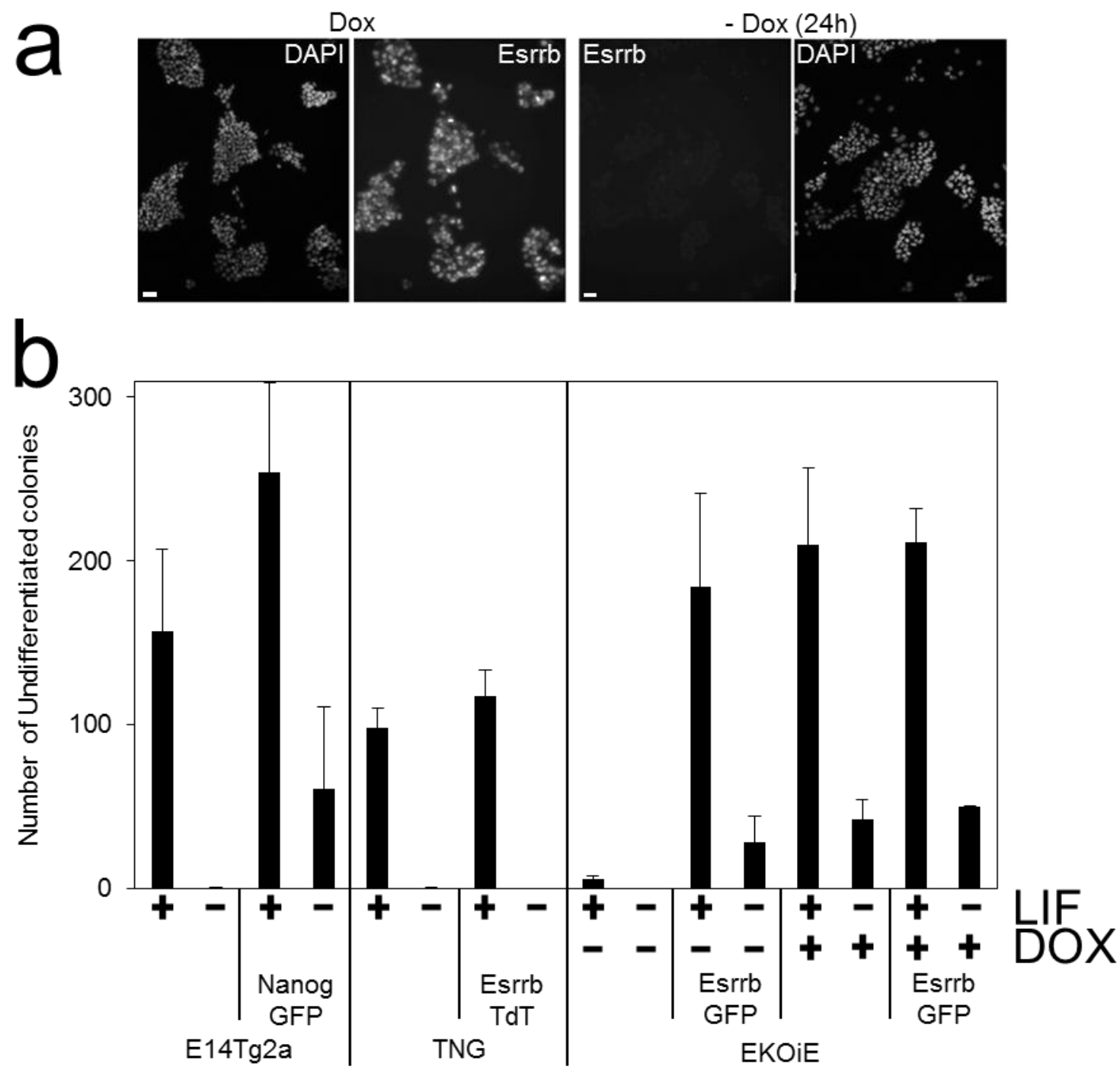
Supplementary Figure 2
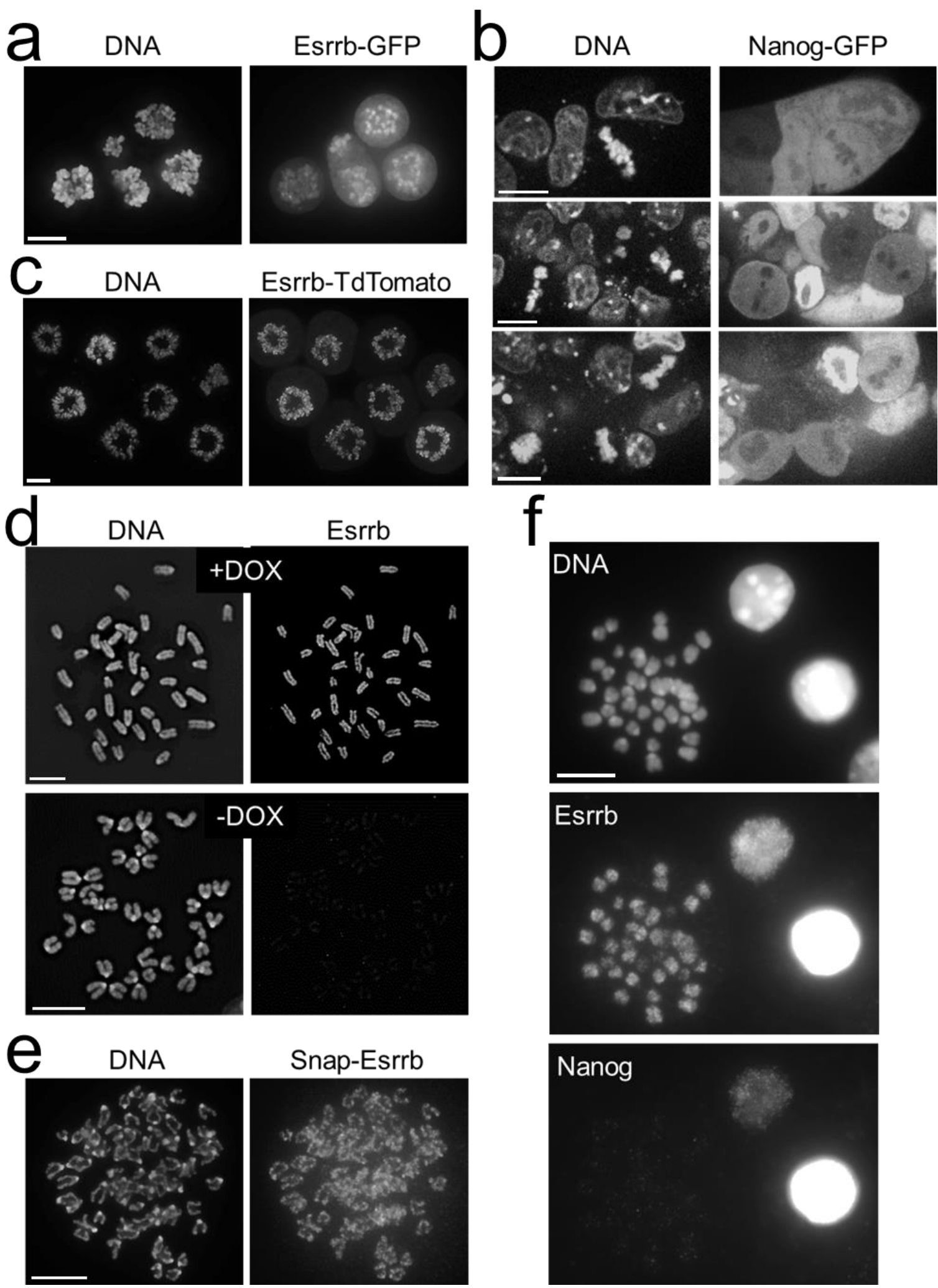

Snap-Esrrb
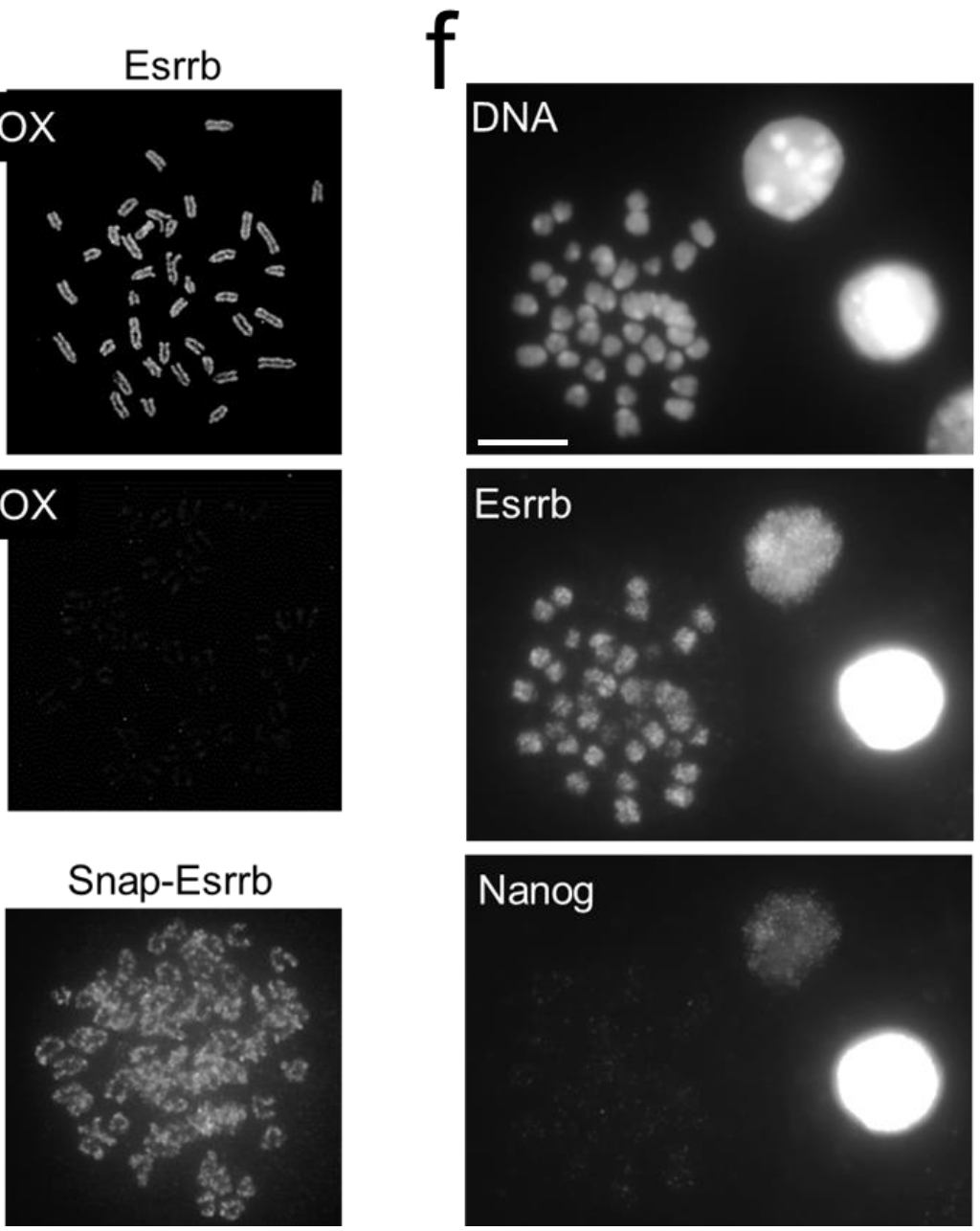


\section{Supplementary Figure 3}

a

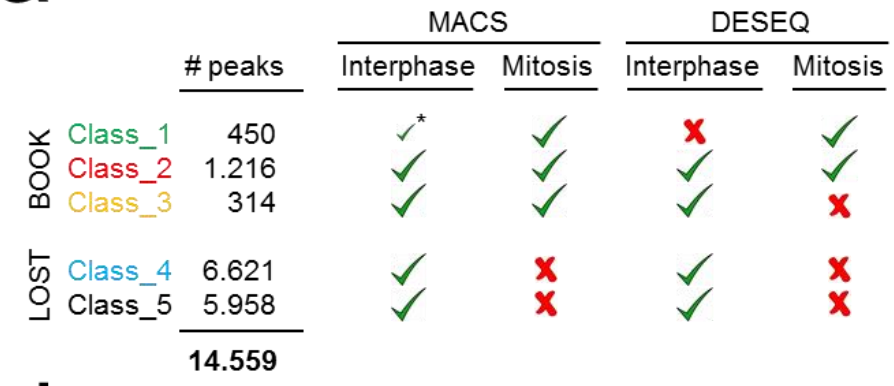

d
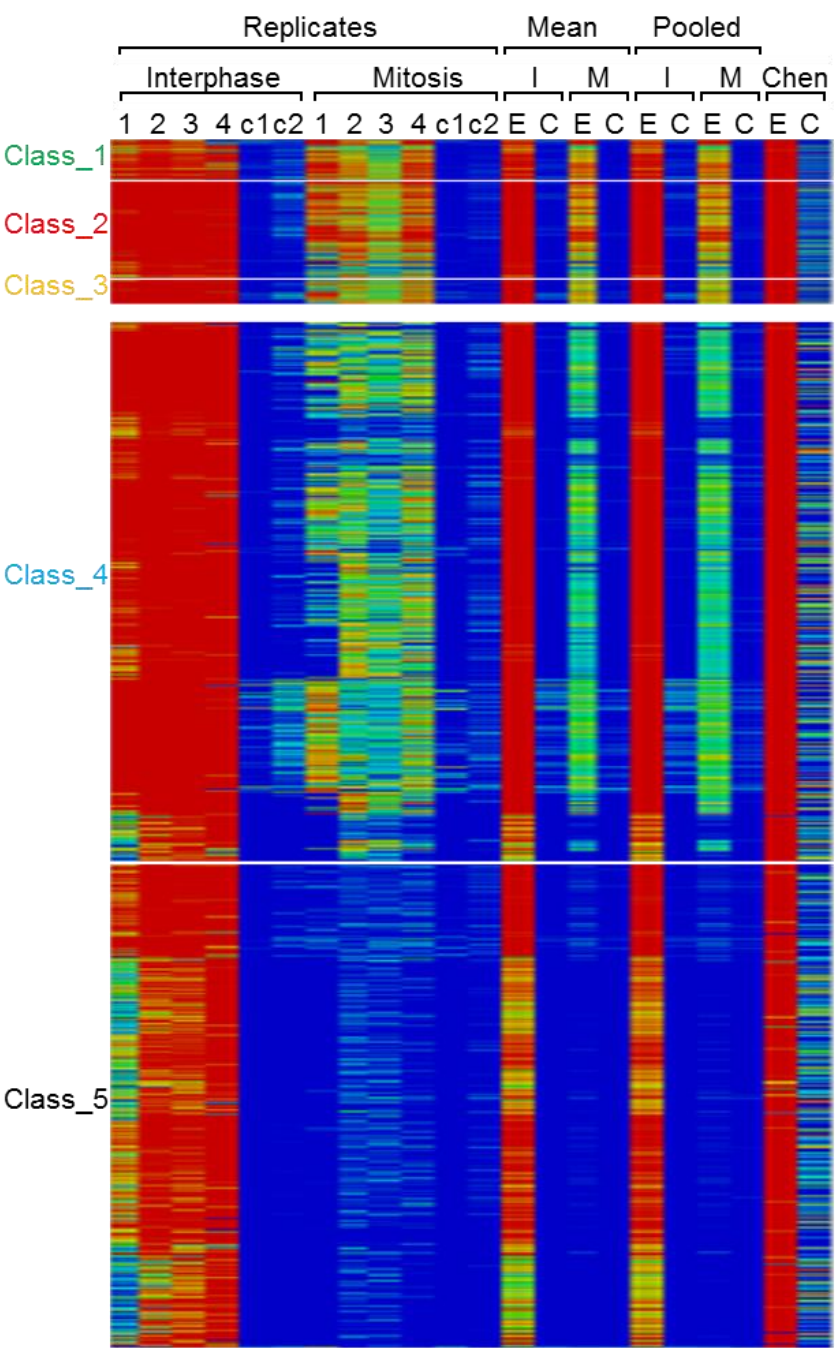

Enrichment

High b

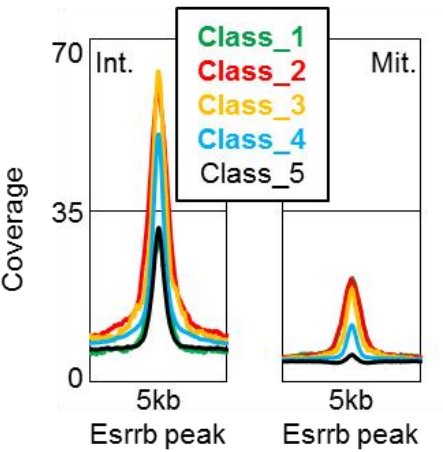

C

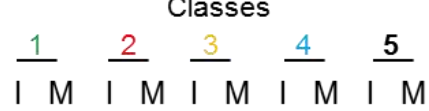

Q

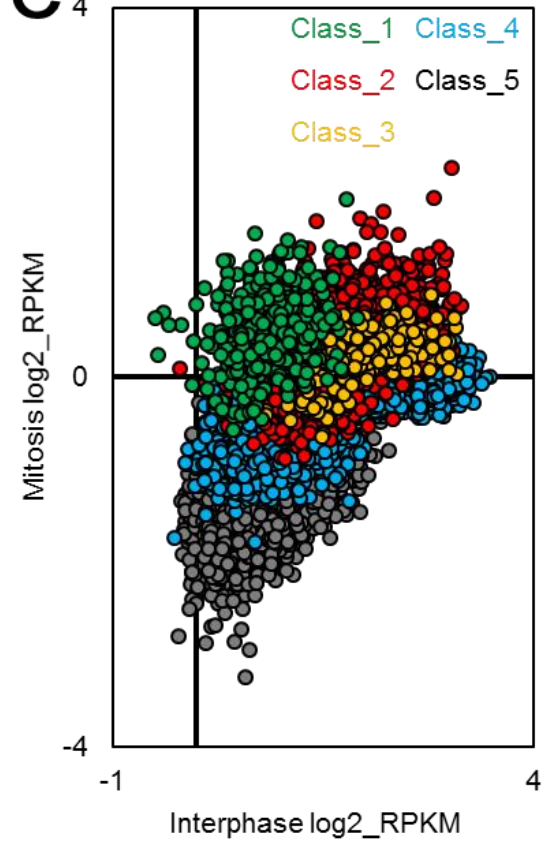




\section{Supplementary Figure 4}

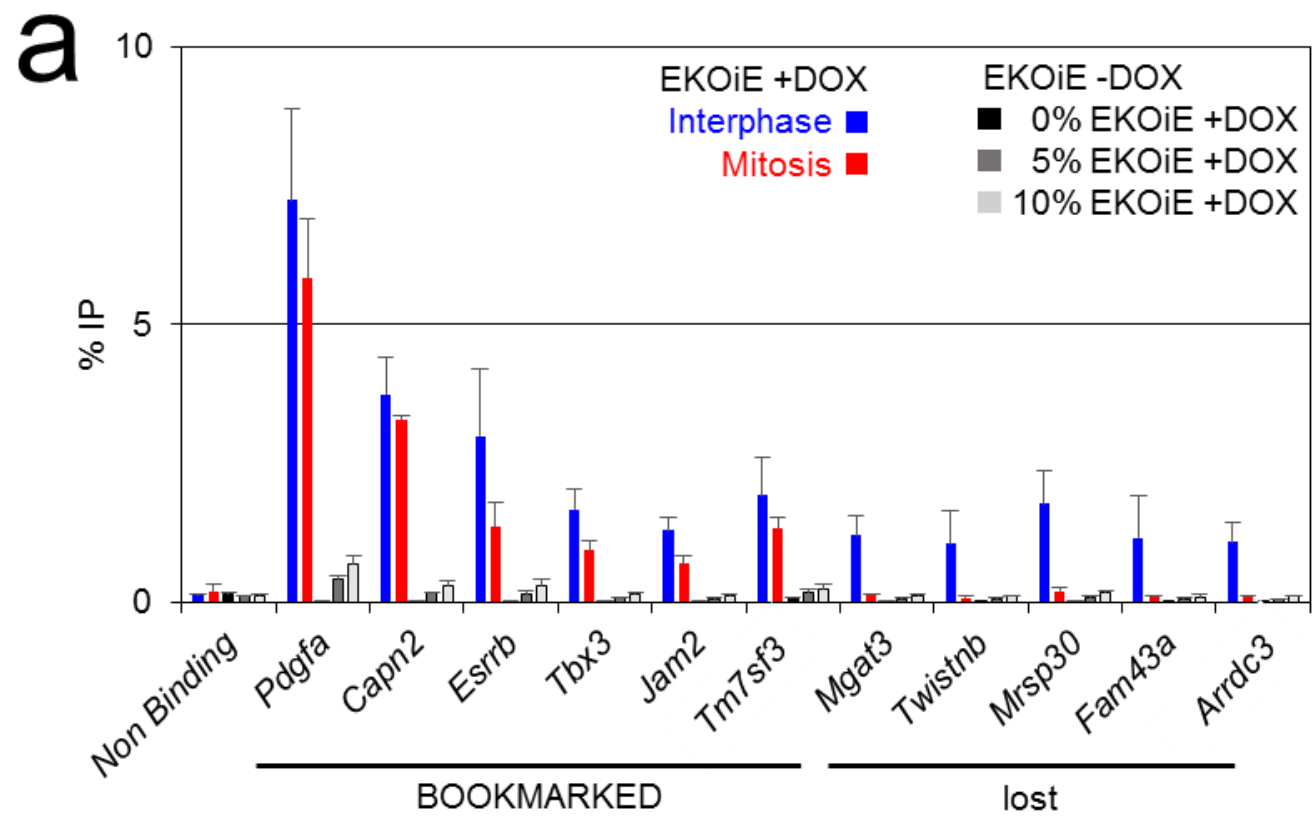

b
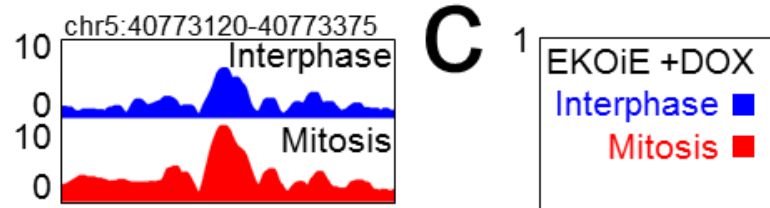

EKOiE -DOX

Interphase

Mitosis

EKOiE +DOX

- $5 \%$ EKOiE +DOX
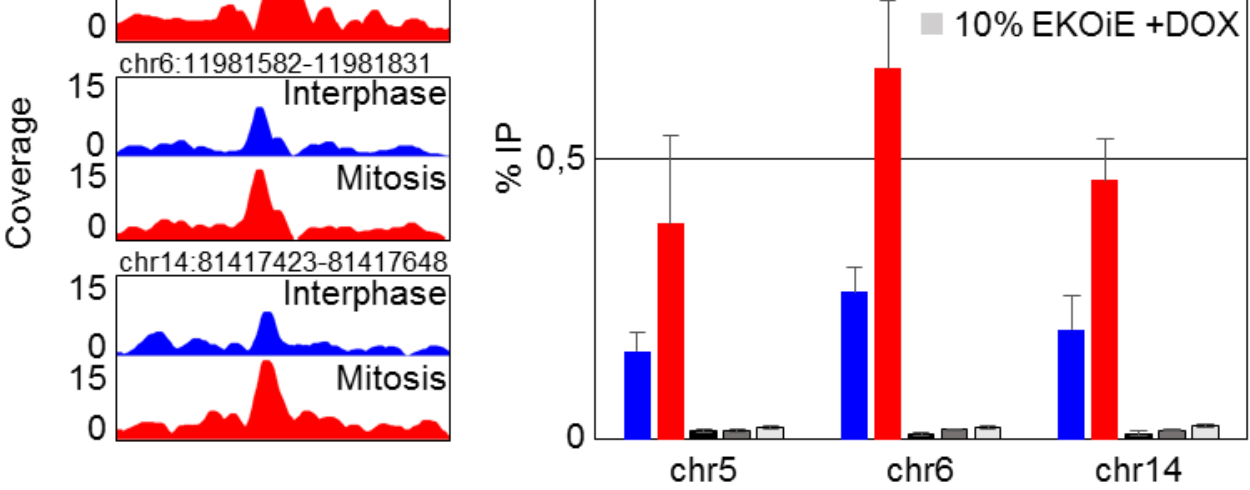


\section{Supplementary Figure 5}

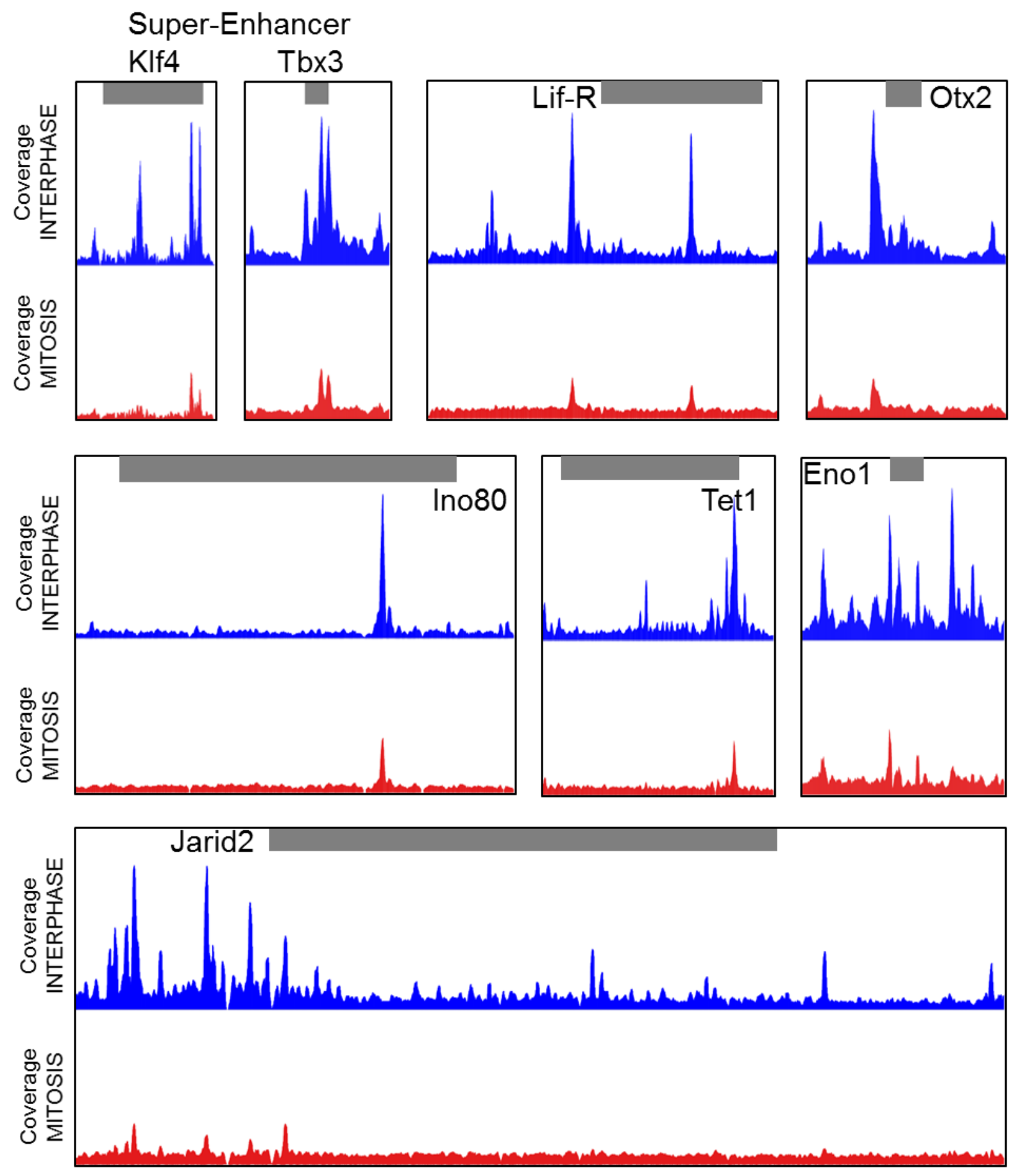




\section{Supplementary Figure 6}

a

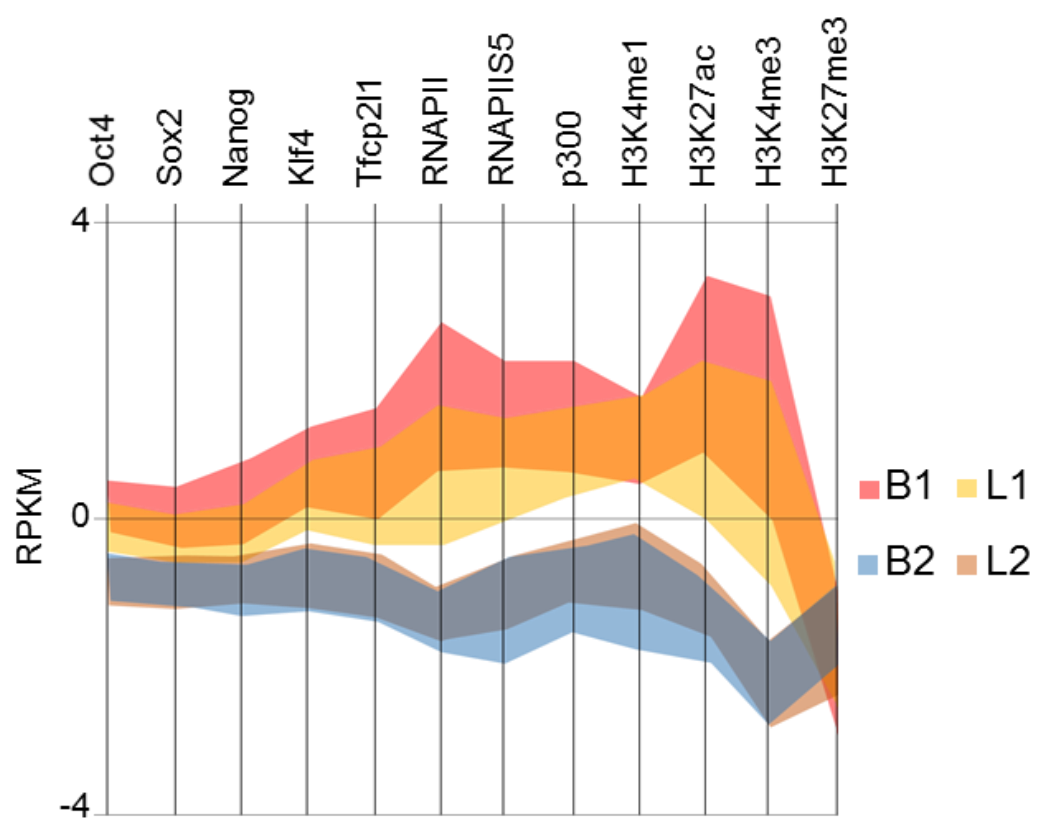

$\mathrm{b}_{\mathrm{B} 1\llcorner 1}$
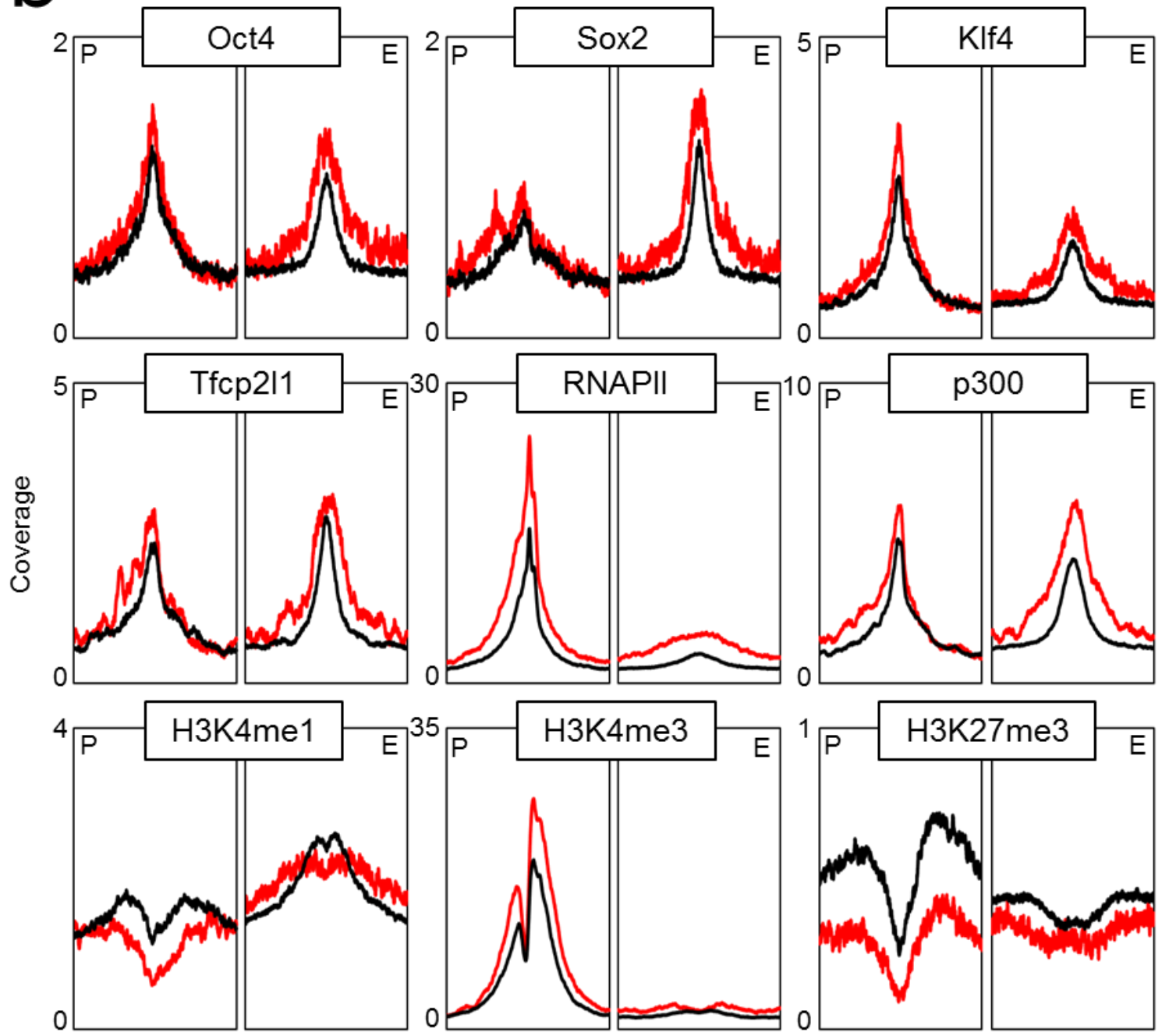

$5 \mathrm{~kb}$ centred on TSS (5'-3' for pronTdtef9 - P) or on Esrrb peak (for enhancers - E) 


\section{Supplementary Figure 7}

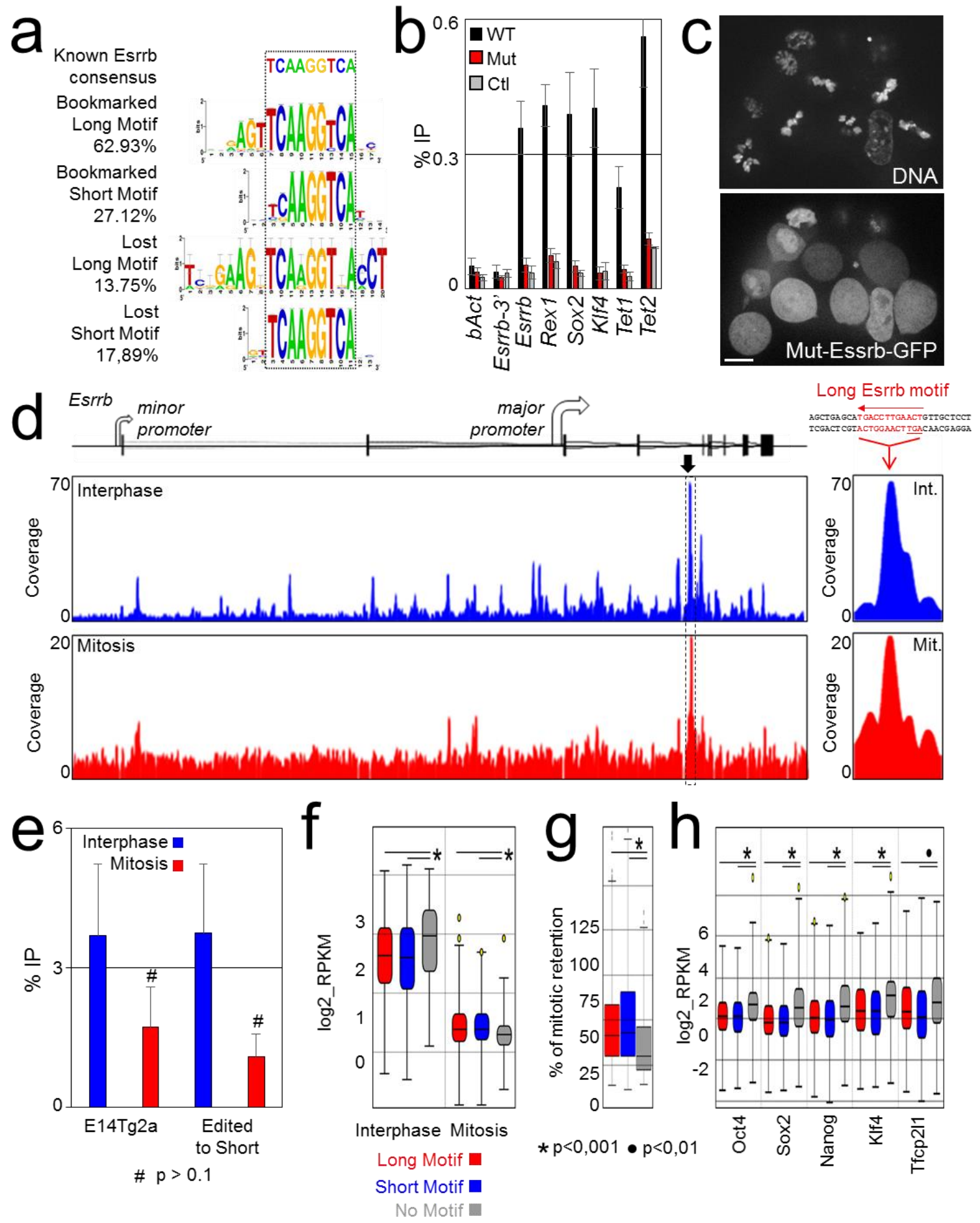




\section{Supplementary Figure 8}
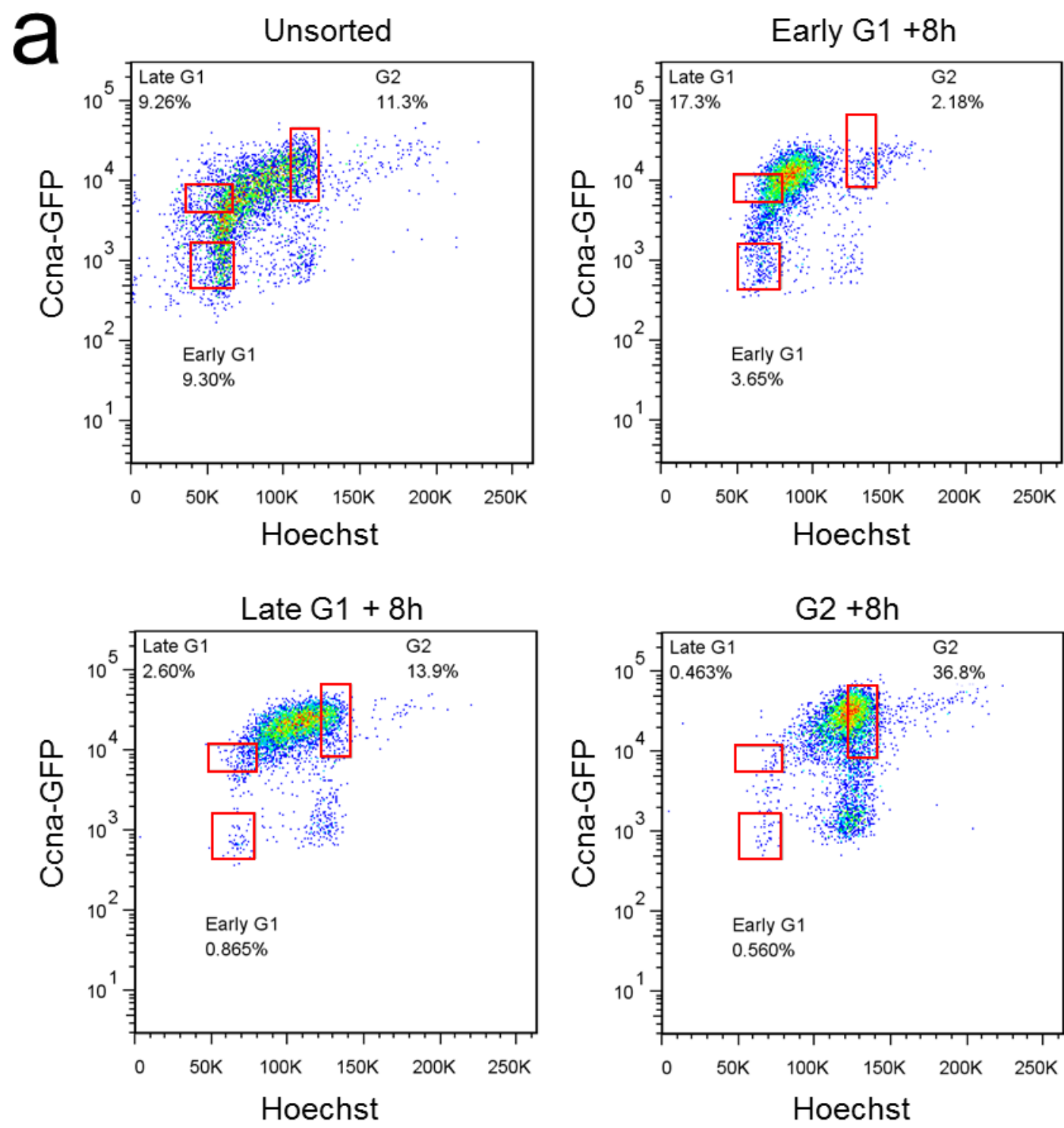

b
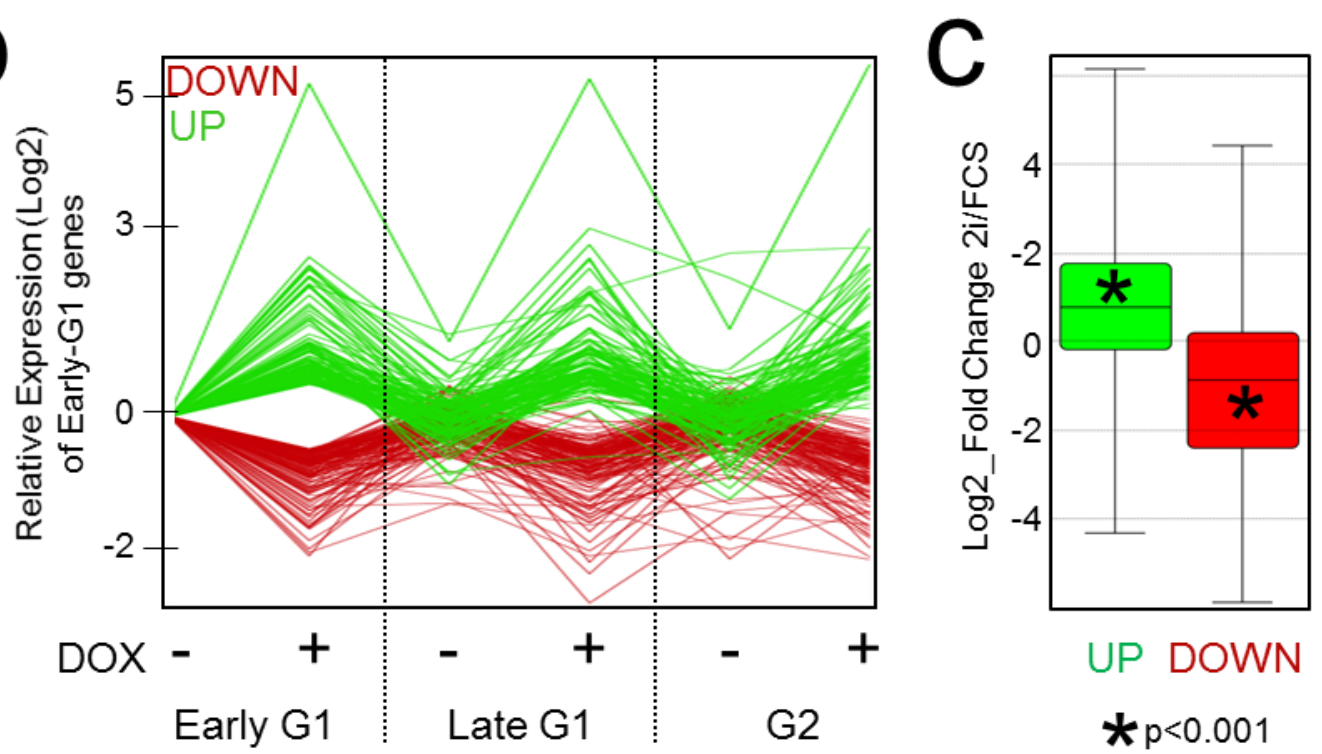